Article

\title{
Density Profiles of 51 Galaxies from Parameter-Free Inverse Models of Their Measured Rotation Curves
}

\author{
Robert E. Criss * and Anne M. Hofmeister \\ Department of Earth and Planetary Science, Washington University, St Louis, MO 63130, USA; \\ hofmeist@wustl.edu \\ * Correspondence: criss@wustl.edu; Tel.: +1-314-935-7440
}

Received: 2 February 2020; Accepted: 24 February 2020; Published: 26 February 2020

\begin{abstract}
Spiral galaxies and their rotation curves have key characteristics of differentially spinning objects. Oblate spheroid shapes are a consequence of spin and reasonably describe galaxies, indicating that their matter is distributed in gravitationally interacting homeoidal shells. Here, previously published equations describing differentially spinning oblate spheroids with radially varying density are applied to 51 galaxies, mostly spirals. A constant volumetric density $\left(\rho, \mathrm{kg} \mathrm{m}^{-3}\right)$ is assumed for each thin homeoid in these formulae, after Newton, which is consistent with RCs being reported simply as a function of equatorial radius $r$. We construct parameter-free inverse models that uniquely specify mass inside any given $r$, and thus directly constrain $\rho$ vs. $r$ solely from velocity $v(r)$ and galactic aspect ratios (assumed as 1:10 for spirals when data are unavailable). Except for their innermost zones, $\rho$ is proven to be closely proportional to $r^{n}$, where the statistical average of $n$ for all 36 spirals studied is $-1.80 \pm 0.40$. Our values for interior densities compare closely with independently measured baryon density in appropriate astronomical environments: for example, calculated $\rho$ at galactic edges agrees with independently estimated $\rho$ of intergalactic media (IGM). Our finding that central densities increase with galaxy size is consistent with behavior exhibited by diverse self-gravitating entities. Our calculated mass distributions are consistent with visible luminosity and require no non-baryonic component.
\end{abstract}

Keywords: inverse models; rotation curves; galactic density; galactic mass; galactic luminosity; Newtonian gravitation; dark matter

\section{Introduction}

Hundreds of astrophysical studies concern the dynamics of galaxies, which are organized assemblies of stars, gas, and dust. Circular or elliptical motions are observed not only in these immense celestial bodies, but also for objects having many other scale lengths. Such rotational motions are classified either as orbits, which are governed by the potential exterior to an object that is often dominated by the effects of external bodies, or as axial spin, which is controlled by the interior potential of the object itself. These two gravitational potentials are mathematically distinct [1-3], matching only on the object's surface [4]. Hence, the induced motions of spins and orbits differ, even though both are more or less circular.

Orbital motions are familiar because many problems (e.g., Keplerian orbits of planets) are accurately described by the reduced 2 body problem, where a large, central mass controls motions of its small satellites, which are assumed to move independently of each other. However, even planetary orbits in our Solar System are not perfectly co-planar, and planet-planet perturbations are well known, which led to the discovery of Neptune. The velocity of such orbital systems projects to infinity at the central point. In great contrast, velocities inside spiral galaxies trend to the null value at the center. 
That the motions of central regions of spiral galaxies resemble spinning records is required for the organized motions of spin $[5,6]$.

Spin is defined as rotation of a body about a special axis where tangential velocity is zero, and that the motions are predicated on interactions of the matter assembling the body itself. These two characteristics distinguish spin from orbits. Spin is quantified by simple equations involving the moment of inertia (I) and angular velocity [7]. Geometry [8] and internal densification affect $I$. For a rigid sphere or spheroid with constant volumetric density $(\rho)$, mass $(M)$, and equatorial body radius $(R), I=2 M R^{2} / 5$. If this rigid spheroid is self-gravitating and conservative, the Virial theorem (VT) provides the tangential equatorial velocity of its spin as:

$$
v_{\text {spin }}^{2}=\frac{3}{2} \frac{G M}{R} \text { at the equatorial perimeter; } v_{\text {spin }}^{2}=0 \text { along the polar axis, }
$$

where $G$ is the gravitational constant [9]. In contrast, orbiting bodies at some distance $r$ from a massive central body such as the Sun are described by:

$$
v^{2}{ }_{\text {orbit }}=\frac{G M_{\text {in }}}{r} \text { for a central point mass or spherical distribution }
$$

Equation (2) was applied in early analyses of galaxies (e.g., [10]). While these equations are deceptively similar, $M$ and $R$ in Equation (1) represent the mass and radius of the object itself, whereas $M_{\text {in }}$ and $r$ in (2) represent the interior mass of the system and the distance of some external object to the system's center of mass. In multi-body systems these differences are extremely important. Clearly, considering orbits (2) rather than spin (1) as describing the coherent rotational motions of the galaxy yields a much larger mass for equivalent values of $r$ and $R$. The poor fit of the orbital model of Equation (2) to rotation curves (Figure 1a) follows and underlies the proposal of dark matter halos [11].

(a)

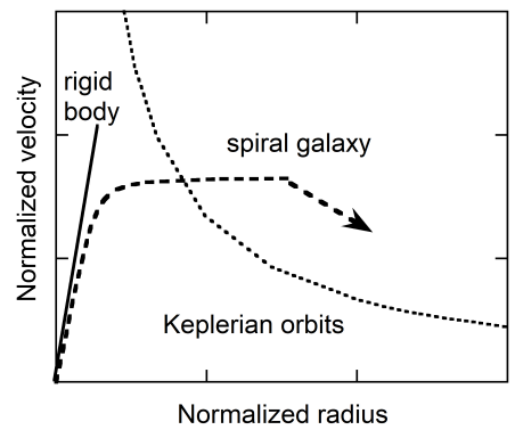

(b)

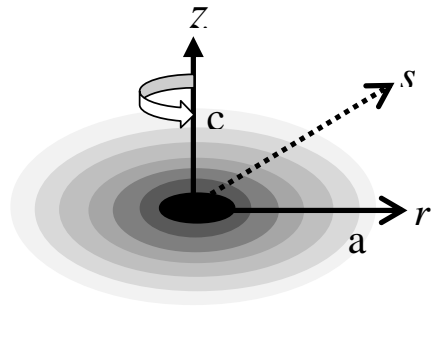

Figure 1. Schematics. (a) Graph comparing spin of a rigid top (with null central velocities) to Keplerian orbits (which have high central velocities) and to galactic RC, which show a gradation between these limiting cases. Mass components cannot be summed to produce RCs [9], as has been pursued in the Newtonian orbital model approach (e.g., [12-14]. (b) Nesting of similar, homogeneous homeoids to form a stratified oblate body, with axes labeled.

Spin is a key attribute of planets and stars, yet this behavior is not predicated on rigidity or high density, because this gravitational phenomenon is described by the geometric arguments of Newton and Laplace [15]. Regarding rigidity, even layers in the dense and highly viscous Earth are decoupled, as demonstrated by westward drift of Earth's outermost lithospheric plates relative to its interior [16]. Decoupling of motions between layers is referred to as differential rotation, and may also exist for Earth's core [17,18]. The precise term is differential spin.

Spin exists regardless of the state of matter, or its density, since spin also describes gas giant planets, rarified stars [19], and many atmospheric phenomena. Hurricanes spin, yet these objects are mostly composed of $\mathrm{N}_{2}$ and $\mathrm{O}_{2}$ gas with embedded water droplets, and so rigidity is not required for spin (e.g., [6]). Galactic rotation curves (RCs), which limit the dependence of velocity $v$ to only equatorial 
radius $r$, implicitly assume zero velocity along the axis of rotation (Figure 1a). This description is consistent with spiral galaxies spinning (Figure 1b).

For spinning, self-gravitating objects, Maclaurin quantitatively showed that the balance between centrifugal and gravitational forces produces an oblate spheroid, as deduced by Newton [20]. This theoretical shape is observationally established for spinning planets and several stars and is confirmed by edge-on images and isophotes of spiral galaxies at wavelengths ranging from the visible to radio, see e.g., Refs. [21-25] and Figure 2. Oblate shapes even describe the rarified, distant material of spirals.

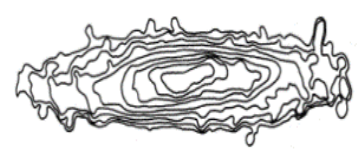

$3109(442 \mathrm{~nm})$

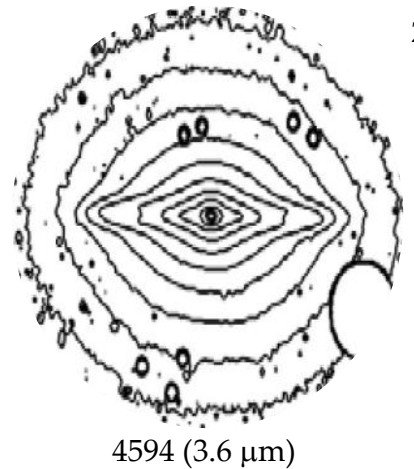

$4594(3.6 \mu \mathrm{m})$

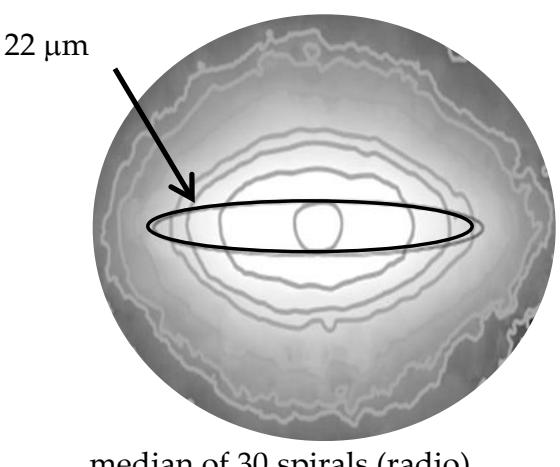

median of 30 spirals (radio)

Figure 2. Isophotes of edge-on galaxies at various wavelengths: (left) New General Catalog (NGG) 3109 in the B-band. Modified after figure 6 in Carignan, C. 1985, Light and mass distribution of the magellanic-type spiral NGC 3109. Astrophys. J. 299, 59-73 [24] with permissions; (middle) NCG 4594 (Sombrero) isophotes at $3.6 \mu \mathrm{m}$ (circles are foreground stars). Modified after figure 1 in Gadotti, D.A. and Sánchez-Janssen, R. 2012, Surprises in image decomposition of edge-on galaxies: Does Sombrero have a (classical) bulge? Mon. Not. R. Astron. Soc. 423, 877-888 [26], with permissions from Oxford University Press on behalf of the R. Astron. Soc.; (right) Grey shades = the L-band from averaging 30 spirals, using the $22 \mu \mathrm{m}$ contour to represent the disk. Modified after figure 6 in [21] (Wiegert, T. and 9 others, 2015. CHANG-ES. IV. Radio continuum emission of 35 edge-on galaxies observed with the Karl G. Jansky very large array in D configuration-Data release 1. Astron. J., 150:81, doi:10.1088/0004-6256/150/3/81), with permissions.

That gaseous astronomical objects hold together during spin is obviated by behavior of planetary and stellar atmospheres. The gas molecules are part of the spin of the star and cannot be treated as each having independent motions. We refer to this behavior as dynamical cohesion, and note that this condition does not require a solid body, but can apply to interacting "particles" comprising a larger, non-solid object, such as cars in congested traffic or droplets in convecting clouds [5,6,27].

Historically, oblate spheroids were recognized as being the required shape for galaxies $[28,29]$. For an oblate spheroid (Figure $1 b$ ), the minor $(c)$ and major $(a)$ axes are tied via the ellipticity $(e)$ :

$$
e=\left(1-c^{2} / a^{2}\right)^{\frac{1}{2}}
$$

This definition for the oblate spheroid surface can be used to relate the axial position $(z)$ to cylindrical radius $(r)$ for each surface of the nested homeoids, because these have the same shape factor $e$ :

$$
z^{2}=\left(1-e^{2}\right)\left(a^{2}-r^{2}\right)
$$

From (4), volumetric density $\rho(z)$ depends on $\rho(r)$, along with $a$ and $e$, for oblate spheroids, which considerably simplifies analyses of RCs from spin.

Given the above, we recently investigated the outer velocities of a spinning oblate spheroid galaxy by applying the rigid body approximation while considering various internal density variations $[5,6]$. Because galaxies are bound systems, their energetics are specified by the Viral theorem (VT) of Clausius. 
Emden's [30] classical book applied the VT to dispersed nebulae and rainclouds. For density governed by a polytrope of index $j$, the mass interior to any given radius is:

$$
M_{\text {in }}=v_{\text {tangent }}^{2} \frac{2}{3}\left(\frac{5-j}{5}\right)^{3} \frac{r}{G} \frac{e}{\arcsin (e)}
$$

Galactic masses calculated from Equation (5) match the luminous mass for the 14 spirals with well-constrained data [5]. Applying Equation (5) with $j=2$, appropriate for mixtures of $\mathrm{H}_{\text {and }} \mathrm{H}_{2}$ gas, to all 356 galaxies with both RC and luminosity data provides a 1:1 trend [6].

Because RC data depict an average condition at an instant of time, the motions must be modeled as being steady-state, i.e., the galaxy is spinning stably. Stability requires that each homeoidal shell (Figure 1b) has constant density. Based on this finding, Criss and Hofmeister [27] addressed differential spin, which allows probing interior motions. Their inverse solution is:

$$
\rho(r)=\frac{1}{6 \pi G}\left(\frac{2 v}{r} \frac{\partial v}{\partial r}+\frac{v^{2}}{r^{2}}\right) \frac{e}{\sqrt{1-e^{2}} \arcsin (e)}
$$

This result was applied to Andromeda but is applicable to isolated galaxies of all morphological types. We made no assumptions other than Newtonian physics and conservation laws. Equation (6) is analytic and exact, has no free parameters, and allows direct and unambiguous extraction of density and mass profiles from RC.

In the present paper, Section 2 describes how spreadsheet data on rotation curves $\left(v_{\text {tangent }}\right.$ as a function of equatorial radius) can be used to compute mass and density as a function of radius, in an approach equivalent to Equation (6). Section 3 applies our model to RC data to directly provide mass and density, each as a function of $r$, for 51 galaxies that range widely in size, RC pattern, and type (spiral, lenticular, irregular, spheroidal, elliptical, and polar ring), although the majority (36) explored are spirals. Although elliptical galaxies and other roundish types do not technically have RC, we include these types because Romanowsky et al. [31] and others have treated their velocity dispersions as RC. We test and verify our approach by comparing our deduced densities with independent measurements involving many astrophysical environments (Table 1). Consistent trends emerge from our analysis and provide the physics underlying empirical luminosity-velocity relations (Section 4). Neither dark matter nor non-Newtonian physics are required to explain galactic rotation curves (Section 5).

\begin{tabular}{|c|c|c|c|}
\hline & & Density & \\
\hline Object or Region & $\mathrm{kg} \mathrm{m}^{-3}$ & $\mathrm{M}_{\text {sun }} \mathrm{pc}^{-3}$ & H Atoms $\mathrm{cm}^{-3}$ \\
\hline Dispersed Solar System ${ }^{1}$ & $5.2 \times 10^{-9}$ & $8 \times 10^{10}$ & $10^{12}$ \\
\hline Molecular Cloud Core & $2 \times 10^{-15}$ & 30,000 & $>10^{6}$ \\
\hline Globular Cluster Core & $7 \times 10^{-18}-10^{-16}$ & $\sim 100-1500$ & $0.4-6 \times 10^{4}$ \\
\hline Best Laboratory Vacuum & $10^{-17}$ & 150 & $6 \times 10^{3}$ \\
\hline Globular Cluster & $\sim 7 \times 10^{-18}$ & $\sim 0.5$ & $\sim 4200$ \\
\hline Giant Molecular Cloud & $\sim 10^{-18}$ & $\sim 15$ & $\sim 600$ \\
\hline Solar Neighborhood ${ }^{2}$ & $\sim 3 \times 10^{-21}$ & $\sim 0.04$ & $\sim 2$ \\
\hline Interstellar Medium ${ }^{3}$ & $\sim 10^{-21}$ & $\sim 0.02$ & $\sim 0.6$ \\
\hline Intergalactic Medium 4 & $\sim 5 \times 10^{-26}$ & $\sim 7 \times 10^{-7}$ & $\sim 3 \times 10^{-5}$ \\
\hline Baryons in Universe 5 & $\sim 10^{-26}$ & $\sim 10^{-7}$ & $\sim 6 \times 10^{-6}$ \\
\hline
\end{tabular}

Table 1. Volumetric densities of different astrophysical environments and objects.

1 Out to the sphere with radius of $30 \mathrm{AU} .{ }^{2}$ From data in Ledrew [32], including dispersed white dwarfs, which agrees with Luyten's [33] estimate. ${ }^{3}$ Ferrière [34] provides $10^{-4}$ to $10^{6}$ atoms per cc for various components in the interstellar medium (ISM). ${ }^{4}$ From Fang et al. [35], the intergalactic media (IGM) is 5 to 200 times denser than the cosmological models of the baryons: we use the average of 100 times. ${ }^{5}$ Cosmological model depending on the Hubble constant [36]. 


\section{Methodology}

Our inverse model is based on the VT, which stems from a mathematical identity. An important consequence for a bound, conservative state is that average linear momentum cancels in all directions over its restricted space [9]. The axial spin of galaxies arising through gravity can thus be analyzed using the VT $[5,6,27]$. Some details are given below. This paper calculates volumetric density.

\subsection{Inverse Models}

Inverse problems are fundamentally different than forward (direct) problems (Table 2). These are best understood via contrast to the latter which are much more familiar. Direct problems are solved by inserting known, or assumed, inputs, such as source characteristics, into a standard equation, formula or program, that returns a result. In contrast, many important problems in astronomy involve the opposite approach, which is to deduce the nature of a remote source from the nature of its output (e.g., [37]). The direct calculation of density profiles from observed RC data, as rendered possible by Equation (6), is a successful example of a solved inverse problem.

Table 2. Comparison of forward and inverse approaches to RC analysis. ${ }^{1}$

\begin{tabular}{cccc}
\hline Type & Input & Physical Model & Output \\
\hline Forward & density and shape ${ }^{2}$ & Newtonian orbits & RC fits \\
Forward & density in a disk-like shape & MOND $^{3}$ & RC fits \\
Inverse & RC and $c / a$ data & Virial theorem & density vs. radius \\
\hline
\end{tabular}

${ }^{1}$ After Groetsch [38] (Chapter 1). ${ }^{2}$ Assumed and iterated until a fit is obtained. ${ }^{3}$ MOdified Newtonian Dynamics includes a parameter in the force law that is varied to obtain a fit to RC data. ${ }^{4}$ Applied to Newton's and Maclaurin's descriptions of spinning, self-gravitating bodies.

Mathematical solutions to inverse problems are not always possible [38], which has made this approach less familiar. The importance of inverse models to astronomy is underscored by Ambartsumian's [39] determination of the proper velocity distribution of stars from observed radial velocity distribution.

\subsection{Homeoid Properties and Their Consistency with Observations}

Diverse astrophysical environments are less dense than vacuums produced in the laboratory (Table 1) and collisions are rare, and so friction is absent, and galaxy motions, shape, and distribution of mass are all gravity driven and linked. Hence, the nested spheroidal shells (Figure 1b) each rotate independently at some angular velocity. Direct evidence for independent rotation of galactic shells is provided by the reduction of tangential velocities above the planes of spiral galaxies [40,41], slower rotation in the bulge than in the disk at any given $r$ in an S0-E6 type [42], and examples of counter rotation and perpendicular rotation (polar rings) in a few spiral galaxies (e.g., [43]).

As Newton discovered, ellipsoidal shells (homeoids) are equipotential [44] (p. 87) and therefore each has a characteristic density. Otherwise, forces along its surface would be unbalanced. Newton's homeoid theorem shows that a test particle inside a homeoid experiences no net force. Constant density for individual homeoids, which is associated with gravitational stability [45,46] (p. 100-101), is appropriate for several reasons. First, $v(r)$ are angular averages that do not describe evolutionary changes. Second, spiral galaxies vary greatly in appearance (star distribution) yet their RC curves are quite similar [47], permitting classification as a very few types (e.g., [48]). Thus, only the radial dependence of the density distribution is important. Third, heterogeneities in the images which correlate with locations of luminous stars do not reveal heterogeneity in density over a larger scale. For example, clouds in Earth's atmosphere are no more dense than dry surrounding air, even though water droplets have high density.

An oblate spheroid is simply a flattened sphere that can be considered to represent a collection of nested homeoids. The self-potential of a spheroid differs from that of a sphere only through 
the ellipticity. Based on Maclaurin's geometrical constraints, Todhunter [20] determined that the self-gravitational potential of a spheroid (Figure $1 b)$ is a simple factor $(k)$ times the self-gravitational potential of a homogenous sphere of equivalent volume, where:

$$
k=\left(1-e^{2}\right)^{1 / 6} \frac{\arcsin (e)}{e} \text { and } U_{g, \text { self }, \text { sphere }}=-\frac{3}{5} \frac{G M_{\text {in }}{ }^{2}}{S}
$$

From Newton's homeoid theorems (e.g., [44]), rotating external homeoids are equipotential surfaces and exert no net force on matter inside, as is the case for spherical shells. Differentiating Equation (7) gives the potential for a homeoid of mass $m$ that surrounds an interior mass $M_{\text {in }}$ :

$$
-U_{\mathrm{g}, \text { homeoid }}=k \frac{G M_{\mathrm{in}} m}{s_{\mathrm{eqv}}}=\frac{G M_{\mathrm{in}} m}{r} \frac{\arcsin (e)}{e}
$$

where the left hand side incorporates the radius of the sphere of equivalent volume for the oblate spheroid of $s_{\text {eqv }}=a\left(1-e^{2}\right)^{1 / 6}$, since the equatorial radius $r$ which varies from 0 to $a$ is most appropriate.

Differentiating the well-known formula for $I$ of an oblate spheroid which is:

$$
I_{\text {oblate }}=\frac{2}{5} M a^{2}=\frac{8 \pi \rho_{\text {ih }}}{15} a^{5}\left(1-e^{2}\right)^{1 / 2}
$$

gives a simple result for the moment of inertia of a homeoid:

$$
I_{\text {homeoid }}=\frac{2}{3} m r^{2}
$$

Existing complicated relationships in Sersic [49] reduce to Equation (10). For reference, homeoid volume $(d V)$ and mass ( $m$, which actually is $d m)$ are, respectively:

$$
d V=4 \pi r^{2} d r\left(1-e^{2}\right)^{1 / 2}=3 V \frac{d r}{r} \text { and } m=\rho d V=3 \rho V \frac{d r}{r}
$$

Applying the Virial theorem to a series of nested coaxial homeoids results in $\left(\mathrm{GM}_{\mathrm{in}} m / r\right) \arcsin (e) / e$ $=2 / 3 m r^{2} \omega^{2}$. Rearranging terms gives the equatorial velocity as:

$$
v_{\text {homeoid }}^{2}=\frac{3 G M_{\text {in }}}{2 r} \frac{\arcsin (e)}{e}
$$

From (12), the angular velocity of any spheroidal shell depends only on its size, ellipticity, and the mass interior to that homeoid, provided that the latter is distributed in a spheroidally symmetric manner (Figure 1b, Figure 2). This relationship, a consequence of Newton's homeoid theorem, differs only from that for a spherical assemblage of particles by a geometric factor involving the ellipticity.

Because $e$ ranges from 0 to 1 , the geometrical factor $\arcsin (e) / e$ ranges only from 1 to $\pi / 2(\sim 1.57)$. Thus, for flattened homeoids, $v^{2}$ is $\sim(2 \pm 0.4) \mathrm{GM}_{\mathrm{in}} / r$, which is not greatly different than the relationship for a spherical shell. Importantly, nested homeoids also spin about the same axis; the composite object is merely a flattened sphere. We reiterate that the shape of the surface of the homeoid shares the ellipticity with the oblate body, per Newton and Laplace.

\subsection{Tie to Previous Conventions and Equations}

The RC literature uses the variable " $r$ " to depict galactic distance in the equatorial plane. The preceding equations used $r$ for cylindrical radius and $s$ for spherical radius where appropriate (see Figure 1b). The remainder of this report uses " $r$ " because we are concerned with the equatorial radii of galaxies.

Calculating orbital velocities of a system of coaxial, rotating spheroidal shells of variable density is straightforward. The relevant mass is the mass of matter interior to a particular shell. 
Note that $n$ in the denominator of equation 15 of Criss and Hofmeister [27] should be replaced by 3. This typo did not affect other equations in [27]. This forward model is not being used here.

According to Marr [50], inverse models for galactic spin which input measured velocities and output mass or density had not been constructed previously. Prior to detailed measurements of RC, Brandt [51] attempted to provide analytical expressions for mass as a function of radius by inverting the formulation for oblate spheroids of Burbidge et al. [29], which describe attraction to the exterior of the oblate $(r>R)$. Setting $e=0$ (spherical symmetry) in their formula and using constant $\rho$ provides a Keplerian orbit, thereby confirming that Burbidge et al. [29] modelled satellite orbits exterior to an oblate body, rather than describing the organized motions inside.

In exploring the case of $e \sim 1$, Brandt [51] arrived at Keplerian orbits in his equation number 25, which instead requires $e=0$. This inconsistency resulted from his inversion including division by the factor $\left(1-e^{2}\right)^{1 / 2}$, which is 0 in the limit of $e=1$. Division by 0 occurred early in Brandt's [51] derivation, specifically in his equation number 7 , which voids his inverse model for orbits about an oblate.

Such difficulties stem partially from the equations for forces and potentials exterior to an oblate body not being cast in particularly useful forms until 2018 [4]. More importantly, correct and simple equations for the gravitational potential and moment of inertia for a homeoid are presented for the first time as equations (8) and (10), above. These new equations are the basis of our inverse model.

\subsection{Methodology for Inverse Modelling}

For spiral galaxies lacking well constrained e, we used $c / a=0.1$ for which $\arcsin (e) / e=1.4871$ (Table 3). These assumed values are consistent with edge-on spirals (excepting Sombrero with its large bulge, Figure 2) and with compiled data on aspect ratios [52], recognizing that tilt makes the objects appear rounder. Moreover, assuming $c / a=0.1$ has little effect, since $\arcsin (e) / e$ neither varies greatly (Table 3), nor has a huge effect, as shown by a few examples in Section 3.

Table 3. Geometrical factors describing galactic shapes.

\begin{tabular}{ccccc}
\hline Shape or Type $^{\mathbf{1}}$ & Object & $\boldsymbol{c}^{\mathbf{1}}$ & Ellipticity & Arcsin $(e) / \boldsymbol{e}$ \\
\hline Sphere & NGC 2434, 4494 & 1 & 0 & 1.0000 \\
E1 & NGC 3379 & 0.98 & 0.2 & 1.0068 \\
cE2, spheroidal & M32; WLM ${ }^{2}$ & 0.87 & 0.49 & 1.0451 \\
S0 & UGC 3993 & 0.75 & 0.66 & 1.0921 \\
E6 & NGC 2768, 4431 & 0.52 & 0.85 & 1.1953 \\
Sombrero & NGC 4594 & 0.4 & 0.917 & 1.2655 \\
Irregular & NGC 3034 & 0.38 & 0.925 & 1.2768 \\
S0/a & NGC 7286 & 0.36 & 0.93 & 1.2843 \\
Flat oblate & All other spirals & 0.1 & 0.995 & 1.4781 \\
Infinite oblate & Limiting value & $\sim 0$ & 1 & 1.5708 \\
\hline
\end{tabular}

${ }^{1}$ Descriptions and data from [23]. ${ }^{2}$ The other 7 dwarf irregulars and spheroidals were assumed to have the same $c / a .{ }^{3}$ We used $c / a=0.1$, based on the infrared contours of Figure 2 that average 30 nearly edge-on spirals [21,22] and the generally accepted view that spirals are thin and flat.

To convert reported values of angular diameter to $\mathrm{kpc}$, we used the average distance in the NASA/IPAC Extragalactic Database (NED) (see Section 2.5). Next, we calculated $M_{\text {in }}$ in a spreadsheet from Equation (12) and then computed $\mathrm{d} M / \mathrm{d} r$. Density is obtained from a geometrical constraint on the homeoids:

$$
\rho(r)=\frac{\partial M}{\partial r} \frac{1}{4 \pi r^{2} c / a}
$$

Results for Andromeda from (6) and (13) are indistinguishable, although the former equation provides the most direct link between density and RC data. 


\subsection{Criteria for Selecting Galaxies and Data Available}

The database on RCs of spiral galaxies is very large, although skewed to SA and SAB classes [53]. In comparison, relatively few irregular, lenticular, elliptical and (rare) polar ring galaxies have been studied, although efforts have been directed towards LSB and dwarf spheroidal galaxies (e.g., [54]). Measurements on non-spiral types concern velocity dispersions, which have been treated as RCs to estimate masses. High resolution, recent studies were sought, particularly for the various morphologic spiral sub-types (a-d), as this sequence illustrates increasing gas content and decreasing bulge size, e.g., [14,55]. As described below, we analyzed 36 spirals and 15 other galaxy types, which encompass known morphologies, cover a wide range of galactic properties, and include both typical and unusual patterns for RC. Ellipticities are listed in Table 3.

Table 4, which lists galaxy properties from [23], is divided into five subsections, which address different issues arising in analyses of RC. Each subsection lists galaxies by NCG number, if available. Types are discussed in [23]. For a consistent measure of size, we used the NED [23] tabulations of distance and of $r$ at $25 \mathrm{~B}$-magnitude $\operatorname{arcsec}^{-2}$ and denote this isophote as the visual edge, for brevity. All distances are based on models which make some assumption about absolute luminosity. For an explanation see [56] (redshift section).

The 1st subsection of Table 4 consists of large and moderate sized spiral galaxies that encompass the main morphologies, have different attributes, and were measured in several studies, mainly due to their proximity (e.g., Andromeda, Triangulum, and Sombrero). In addition, the Milky Way provides a unique internal view of rotational data [57-60]. NGC 253 and 2599 have declines in $v$ with $r$ at large $r$ [61]. Large Uppsala General Catalog (UGC) 2855 has data extending far beyond its visible disk [62]. Irregular NGC 3034 has lopsided rotation curves [63]. This galaxy is similar in size to NGC 4826, which counter-rotates, and to NGC 7793 [54].

The 2nd subsection consists of galaxies considered by Bottema and Pestaña [64] to have highly desirable characteristics for accurate analysis. Useful selection criteria include a well-established distance, symmetric RCs that extend beyond the optical disk, inclinations between 50 and $80^{\circ}$, and a range of masses. We omitted 2 of the 12 galaxies from [64] because their rotation curves are similar to others in this grouping and/or have widely spaced data points. Of the 10 galaxies examined, 8 are spirals, NGC 4789a is irregular, and NGC 1560 has low surface brightness.

The 3rd subsection lists all Messier objects studied by Sofue and collaborators using tabular data on Sofue's website [65]. Not only are various types of spirals included, but the method of data analysis is consistent, and both centers of the galaxies and outer reaches were examined and merged in a consistent fashion. Sofue et al's $[53,66]$ measurements compare reasonably well with more recent studies [14] and have been used to evaluate models [67].

The 4th and 5th subsections cover galactic types other than spirals. We used high-resolution data or extended RC, if available, but for types such as ellipticals and spheroidals, velocity dispersion curves are the only data available (e.g., [68]).

Table 4. List of studied galaxies with characteristics from the NASA/IPAC Extragalactic Database (NED [23]) and references for RC data.

\begin{tabular}{|c|c|c|c|c|c|c|c|c|}
\hline NGC & Other Name & Type & Notes & Refs. & $\begin{array}{c}\text { Distance } \\
\text { (Mpc) }\end{array}$ & $\begin{array}{c}L_{\text {vis }} \\
\left(L_{\text {Sun,vis }}\right)\end{array}$ & $\begin{array}{c}L_{21}{ }^{1} \\
\left(L_{\text {Sun,21 }}\right)\end{array}$ & $\begin{array}{c}\text { Edge }^{2} \\
\text { (kpc) }\end{array}$ \\
\hline \multicolumn{9}{|c|}{ Well-Studied Spirals } \\
\hline - & Milky Way & SBC & inside view & {$[57,58]$} & & $4 \times 10^{10}$ & & 18 \\
\hline 224 & Andromeda & $\mathrm{SA}(\mathrm{s}) \mathrm{b}$ & nearby, bulge & [59] & 0.78 & $3.19 \times 10^{10}$ & 2300 & 23.3 \\
\hline 253 & & $\mathrm{SAB}(\mathrm{s}) \mathrm{c}$ & starburst & [69] & 3.16 & $1.88 \times 10^{10}$ & & 18 \\
\hline 598 & Triangulum & $\mathrm{SA}(\mathrm{s}) \mathrm{cd}$ & no bulge & {$[14,70]$} & 0.84 & $3.65 \times 10^{9}$ & 1110 & 9.45 \\
\hline 925 & & $\mathrm{SAB}(\mathrm{s}) \mathrm{d}$ & large $\mathrm{H}$ cloud & [54] & 8.6 & $6.50 \times 10^{9}$ & 2830 & 16.2 \\
\hline 2599 & UGC 4458 & SAa & high velocity & [61] & 64.2 & $5.01 \times 10^{10}$ & 13,800 & 25.8 \\
\hline 3034 & M82 & I0, edge-on ${ }^{3}$ & starburst & [63] & 4.01 & $8.81 \times 10^{9}$ & & 7.6 \\
\hline 4594 & Sombrero & $\mathrm{SA}(\mathrm{s}) \mathrm{a}$ & huge bulge & {$[71,72]$} & 10.0 & $6.10 \times 10^{10}$ & & 14.4 \\
\hline
\end{tabular}


Table 4. Cont.

\begin{tabular}{|c|c|c|c|c|c|c|c|c|}
\hline NGC & Other Name & Type & Notes & Refs. & $\begin{array}{c}\text { Distance } \\
\text { (Mpc) }\end{array}$ & $\begin{array}{c}L_{\text {vis }} \\
\left(L_{\text {Sun,vis }}\right)\end{array}$ & $\begin{array}{c}L_{21}{ }^{1} \\
\left(L_{\text {Sun,21 }}\right)\end{array}$ & $\begin{array}{c}\text { Edge }^{2} \\
(\mathrm{kpc})\end{array}$ \\
\hline 4826 & M64-Evil Eye & (R)SA(rs)ab & counter rotating & [54] & 5.16 & $1.60 \times 10^{10}$ & & 7.8 \\
\hline 7793 & & $\mathrm{SA}(\mathrm{s}) \mathrm{d}$ & jets, bulge & [54] & 3.93 & $3.70 \times 10^{9}$ & 739 & 8.0 \\
\hline- & UGC 2855 & $\mathrm{SABC}$ & distant & {$[62,67]$} & 79 & $9.90 \times 10^{10}$ & & 15.8 \\
\hline \multicolumn{9}{|c|}{ Spirals with Desirable Characteristics } \\
\hline 1560 & & $\mathrm{SA}(\mathrm{s}) \mathrm{d}$ & LSB, edge on & [64] & 3.28 & $7.80 \times 10^{8}$ & 21.5 & 5.56 \\
\hline 2403 & & $\mathrm{SAB}(\mathrm{s}) \mathrm{cd}$ & bright in HII & [66] & 3.48 & $4.81 \times 10^{9}$ & & 14.15 \\
\hline 2841 & & $\mathrm{SA}(\mathrm{r}) \mathrm{b}$ & LINER, bulge & [66] & 17.6 & $7.43 \times 10^{10}$ & & 20.73 \\
\hline 2903 & & $\mathrm{SAB}(\mathrm{rs}) \mathrm{bc}$ & starburst & [66] & 8.70 & $2.15 \times 10^{10}$ & & 16.8 \\
\hline 3109 & & $\mathrm{SB}(\mathrm{s}) \mathrm{m}$ & small, edge on & [64] & 1.33 & $2.51 \times 10^{8}$ & & 6.35 \\
\hline 3198 & & $\mathrm{SB}(\mathrm{rs}) \mathrm{C}$ & psuedobulge & [65] & 14.0 & $1.28 \times 10^{10}$ & & 20.3 \\
\hline $4789 a$ & DDO 154 & $\mathrm{IB}(\mathrm{s}) \mathrm{m}$ & LSB, dwarf & [64] & 3.84 & $4.40 \times 10^{7}$ & & 1.72 \\
\hline 5585 & & $\mathrm{SAB}(\mathrm{s}) \mathrm{d}$ & psuedobulge & [64] & 8.0 & $1.71 \times 10^{9}$ & 277 & 7.07 \\
\hline 6503 & & $\mathrm{SA}(\mathrm{s}) \mathrm{cd}$ & LINER & [64] & 5.52 & $2.42 \times 10^{9}$ & & 6.42 \\
\hline 7331 & & $\mathrm{SA}(\mathrm{s}) \mathrm{b}$ & bulge & [66] & 14.3 & $5.32 \times 10^{10}$ & 28,300 & 23.84 \\
\hline \multicolumn{9}{|c|}{ Messier Spirals } \\
\hline 1068 & M77 & (R)SA(rs)b & Seyfert & [66] & 12.65 & $2.94 \times 10^{10}$ & & 16.5 \\
\hline 3031 & M81 & $\mathrm{SA}(\mathrm{s}) \mathrm{ab}$ & grand design & [66] & 3.71 & $2.04 \times 10^{10}$ & & 14.7 \\
\hline 4192 & M98 & $\mathrm{SAB}(\mathrm{s}) \mathrm{ab}$ & active nucleus & [53] & 15.61 & $2.05 \times 10^{10}$ & 8620 & 23.8 \\
\hline 4254 & M99 & $\mathrm{SA}(\mathrm{s}) \mathrm{c}$ & star forming & [53] & 15.43 & $2.44 \times 10^{10}$ & & 13.5 \\
\hline 4258 & M106 & $\mathrm{SAB}(\mathrm{rs}) \mathrm{bc}$ & LINER & [66] & 7.46 & $1.87 \times 10^{10}$ & & 23.8 \\
\hline 4303 & M61 & $\mathrm{SAB}(\mathrm{rs}) \mathrm{bc}$ & supernovae & [66] & 12.0 & $1.83 \times 10^{10}$ & & 14.5 \\
\hline 4321 & M100 & $\mathrm{SAB}(\mathrm{s}) \mathrm{bc}$ & grand design & [66] & 16.56 & $4.46 \times 10^{10}$ & & 21.0 \\
\hline 4501 & M88 & $\mathrm{SA}(\mathrm{rs}) \mathrm{b}$ & Seyfert & [53] & 18.79 & $6.50 \times 10^{10}$ & & 19.3 \\
\hline 4548 & M91 & $\mathrm{SB}(\mathrm{rs}) \mathrm{b}$ & LINER & [53] & 16.36 & $2.78 \times 10^{10}$ & & 13.1 \\
\hline 4569 & M90 & $\mathrm{SAB}(\mathrm{rs}) \mathrm{ab}$ & LINER & [66] & 12.29 & $1.93 \times 10^{10}$ & & 17.0 \\
\hline 4736 & M94 & (R)SA(r)ab & LINER, ring & [66] & 5.02 & $9.85 \times 10^{9}$ & & 10.2 \\
\hline 5055 & M63 Sunflower & $\mathrm{SA}(\mathrm{rs}) \mathrm{bc}$ & flocculent & [66] & 8.0 & $1.73 \times 10^{10}$ & & 17.5 \\
\hline 5194 & M51 Whirlpool & $\mathrm{SABC}$ & has companion & [66] & 7.85 & $2.14 \times 10^{10}$ & & 12.8 \\
\hline 5236 & M83 & $\mathrm{SAB}(\mathrm{s}) \mathrm{c}$ & two nuclei & [66] & 4.8 & $3.20 \times 10^{10}$ & & 19.0 \\
\hline 5457 & M101 Pinwheel & $\mathrm{SAB}(\mathrm{rs}) \mathrm{cd}$ & star forming & [66] & 6.81 & $2.22 \times 10^{10}$ & & 27.3 \\
\hline \multicolumn{9}{|c|}{ Dwarf Irregular and Spheroidal Galaxies } \\
\hline- & WLM & $\mathrm{Ib}(\mathrm{s}) \mathrm{m}$ & isolated LSB & [73] & 0.985 & $5.83 \times 10^{7}$ & & 1.8 \\
\hline- & $\mathrm{M} 81 \mathrm{dWb}$ & $\operatorname{Im}$ & star forming & {$[74]$} & 7.66 & $7.13 \times 10^{7}$ & & 1.45 \\
\hline- & Holmberg II & $\operatorname{Im}$ & star forming & [74] & 3.30 & $4.70 \times 10^{8}$ & 92.4 & 4.43 \\
\hline - & Carina & $\mathrm{dSph}, \mathrm{dE} 3$ & classical dSph & [75-78] & 0.103 & $2.95 \times 10^{5}$ & & 0.45 \\
\hline - & Draco & dSph, Epec & classical dSph & [75-78] & 0.082 & $2.95 \times 10^{5}$ & & 0.60 \\
\hline - & Fornax & $\mathrm{dSph}, \mathrm{dE4}$ & classical dSph & [75-78] & 0.139 & $1.30 \times 10^{7}$ & & 1.42 \\
\hline- & Leo I & $\mathrm{dSph}, \mathrm{dE} 3$ & classical dSph & [75-78] & 0.246 & $4.99 \times 10^{6}$ & & 0.43 \\
\hline \multicolumn{9}{|c|}{ Lenticular and Elliptical Galaxies } \\
\hline - & UGC3993 & $\mathrm{S} 0 ?$ & bulge & {$[61]$} & 62 & $1.21 \times 10^{10}$ & & 15 \\
\hline 7286 & UGC12043 & $\mathrm{S} 0 / \mathrm{a}$ & no bulge & [61] & 20.5 & $2.45 \times 10^{9}$ & & 5.6 \\
\hline 2768 & & S0-E6 edge & Seyfert, jets & [42] & 19.3 & $2.80 \times 10^{10}$ & 2570 & 22.8 \\
\hline 3379 & M105 & E1 & elliptical & [31] & 11.0 & $2.11 \times 10^{10}$ & 138 & 8.5 \\
\hline 2434 & & E0-1 & elliptical & [68] & 26.9 & $2.38 \times 10^{10}$ & & 15.4 \\
\hline 221 & M32 & $\mathrm{cE} 2$ & compact dwarf & [55] & 0.775 & $2.61 \times 10^{8}$ & 0.183 & 1.0 \\
\hline 4431 & VCC1010 & $\mathrm{dE}, \mathrm{SA} 0(\mathrm{r})$ & dwarf elliptical & [79] & 16 & $9.87 \times 10^{8}$ & & 4.06 \\
\hline \multicolumn{9}{|c|}{ Polar Ring or Disk Galaxies } \\
\hline $4560 \mathrm{~A}$ & The prototype & S0/a pec? & $2 \perp$ disks & [80] & 46.4 & $9.67 \times 10^{9}$ & & 16.5 \\
\hline
\end{tabular}

\section{Results: Mass and Density from Inverse Models of Rotation Curves}

Most of the visualizations of mass and density for the individual galaxies are placed in Appendix A. Results are summarized in Table 4: Note: this broadside and after the references.

\subsection{Detailed Analysis of the Milky Way}

Sofue [58] compiled available data on the Milky Way to provide a RC from $0.001 \mathrm{kpc}$ to the outer reaches, using the accepted value of $v=200 \mathrm{~km} \mathrm{~s}^{-1}$ near the Sun. Recent observations at greater distances [81,82] motivated revision of the outer RCs to higher velocities [59]. We combine $v$ at low $r$ from Sofue's [58] compilation with $v$ at high $r$ from his [59] compilation and use the differences in values to gauge uncertainties.

Assuming $c / a=1,0.5,0.1$ or 0.01 causes the calculated values for $M_{\mathrm{in}}$ to vary only by a factor of $\sim 1.5$ (Figure 3a). Since $e$ can vary only from 0 to 1 , this range encompasses all reasonable values for $e$. Calculated $M_{\text {in }}$ values for flat shapes (c/a $=0.1$ or 0.01$)$ are similar at any $r$. Due to this finding and 
the behavior observed for density (discussed below), we use the nominal aspect ratio $(c / a=0.1)$ for most spiral galaxies unless data exist (Table 3), permitting us to concentrate on the effects of different patterns for RC.
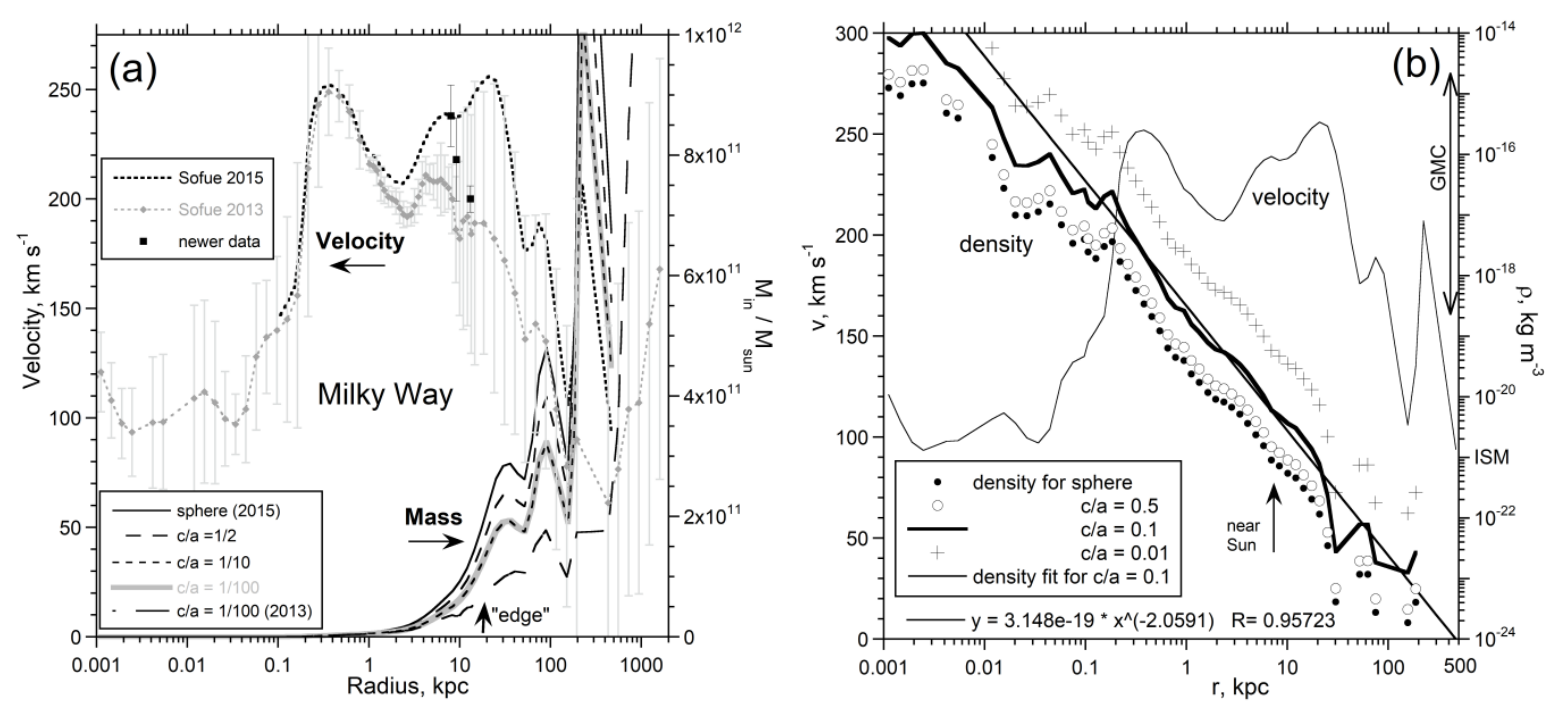

Figure 3. Inverse models showing the effect of varying $c / a$ over a large range for an internally probed galaxy, the Milky Way. Compiled RCs are used to calculate (a) mass inside and (b) density at any given radius. In part (a), upper box describes line patterns associated with RCs $[58,59,66]$ using the left axis and two additional constraints on $v$ near the Sun $[81,82]$ and from globular cluster data [60]. Lower box lists aspect ratios assumed in analyzing each dataset for mass, shown on the right axis. Error bars on $v$ from [58] are shown. Downturns in $M_{\text {in }}$ and $\rho$ indicate that the "edge" of the Galaxy is gradual and between 18 and $30 \mathrm{kpc}$. In (b), density above $r=0.1 \mathrm{kpc}$ was fit to a power law, as shown.

Notably, if $v$ decreases weakly as $r$ increases, calculated values for $M_{\text {in }}$ increase with $r$, as is required by geometry. This behavior largely arises because volume is $a^{3}$ times an ellipticity factor for a spheroid. For a strong decrease in $v$, the calculated values of $M_{\text {in }}$ decrease with $r$. This unrealistic behavior partially results from large uncertainties in $v$ measured near the termination of the Milky Way, as shown in Figure 3a. If the edge were sharp, $M_{\text {in }}$ would increase with $r$ up to some radius, and remain constant thereafter. Our results indicate that the galactic edge is not sharp, but grades into the IGM, which is consistent with the known existence of substantial outlying material, such as gas, globular clusters, or satellite galaxies [83,84].

Most of the mass in the Milky Way (MW) lies at $r$ beyond the visible disk at $18 \mathrm{kpc}$. This behavior occurs because spheroid volume is $a^{3}$ times an ellipticity factor and underlies velocity profiles being flat at large $r$. Luminosity from visible wavelengths is therefore derived from a much smaller mass, mainly that inside the visible edge (Tables 4 and 5). 
Table 5. Properties of galaxies extracted from rotation curves, with a focus on volumetric density.

\begin{tabular}{|c|c|c|c|c|c|c|c|c|c|c|}
\hline \multicolumn{11}{|c|}{ Visible Edge Properties } \\
\hline Name & $\begin{array}{c}M_{\text {in }} \\
\left(M_{\text {Sun }}\right)\end{array}$ & $\begin{array}{c}L_{\text {vis }} / M_{\text {in }} \\
\left(L_{\text {Sun }} / M_{\text {Sun }}\right)\end{array}$ & $\begin{array}{c}\text { Density } \\
\left(\mathrm{kg} \mathrm{m}^{-3}\right)\end{array}$ & $\begin{array}{c}r_{\text {turndown }} \\
(\mathbf{k p c})\end{array}$ & $\begin{array}{l}\text { Lowest } \rho \\
\left(\mathrm{kg} \mathrm{m}^{-3}\right) \\
\end{array}$ & $\begin{array}{c}\rho \text { at } 0.1 \mathrm{kpc} \\
\left(\mathrm{kg} \mathrm{m}^{-3}\right)\end{array}$ & $\begin{array}{c}\text { Highest } \rho \\
\left(\mathrm{kg} \mathrm{m}^{-3}\right)\end{array}$ & Power $^{1}$ & $\begin{array}{l}r_{\max } \\
(\mathbf{k p c})\end{array}$ & $\begin{array}{c}M_{\max } \\
\left(M_{\text {Sun }}\right)\end{array}$ \\
\hline \multicolumn{11}{|c|}{ Large Well-Studied Spirals } \\
\hline Milky Way & $1.21 \times 10^{11}$ & 0.330 & $1.30 \times 10^{-21}$ & $18-30$ & $1.0 \times 10^{-24}$ & $1.00 \times 10^{-17}$ & $1.00 \times 10^{-14}$ & -2.06 & 230 & $1.03 \times 10^{12}$ \\
\hline 224 & $1.44 \times 10^{11}$ & 0.222 & $4.20 \times 10^{-22}$ & $?$ & $2.0 \times 10^{-24}$ & $4.00 \times 10^{-17}$ & $4.00 \times 10^{-17}$ & -2.11 & 285 & $7.03 \times 10^{11}$ \\
\hline 253 & $4.30 \times 10^{10}$ & 0.437 & $1.00 \times 10^{-21}$ & $?$ & $2.8 \times 10^{-22}$ & $9.00 \times 10^{-18}$ & $7.00 \times 10^{-17}$ & $-1.5,-2.0$ & 12.68 & $6.90 \times 10^{10}$ \\
\hline 598 & $1.52 \times 10^{10}$ & 0.240 & $1.30 \times 10^{-21}$ & $?$ & $8.0 \times 10^{-22}$ & $1.80 \times 10^{-17}$ & $1.80 \times 10^{-17}$ & -1.57 & 15 & $2.60 \times 10^{10}$ \\
\hline 925 & $2.00 \times 10^{10}$ & 0.325 & $8.60 \times 10^{-22}$ & 13 & $8.6 \times 10^{-22}$ & & $3.00 \times 10^{-20}$ & -0.9 & 13 & $2.03 \times 10^{10}$ \\
\hline 2599 & $1.90 \times 10^{11}$ & 0.264 & $3.51 \times 10^{-22}$ & $20 ?$ & $3.1 \times 10^{-23}$ & $3.54 \times 10^{-17}$ & $3.54 \times 10^{-17}$ & -2.36 & 56 & $3.52 \times 10^{11}$ \\
\hline 3034 & $1.00 \times 10^{10}$ & 0.881 & $2.20 \times 10^{-21}$ & $?$ & $3.0 \times 10^{-21}$ & $3.70 \times 10^{-18}$ & $1.00 \times 10^{-16}$ & $-1.5,-1.9$ & 3.57 & $7.20 \times 10^{9}$ \\
\hline 4594 & $2.00 \times 10^{11}$ & 0.305 & $3.30 \times 10^{-21}$ & $?$ & $3.0 \times 10^{-21}$ & $8.70 \times 10^{-18}$ & $1.60 \times 10^{-15}$ & -1.6 & 9.4 & $1.58 \times 10^{11}$ \\
\hline 4826 & $1.90 \times 10^{10}$ & 0.842 & $4.50 \times 10^{-21}$ & 20 & $1.0 \times 10^{-22}$ & $1.00 \times 10^{-17}$ & $2.00 \times 10^{-18}$ & -1.92 & 21.7 & $5.69 \times 10^{10}$ \\
\hline 7793 & $8.80 \times 10^{9}$ & 0.421 & $1.20 \times 10^{-21}$ & $6-7$ & $5.0 \times 10^{-23}$ & $6.40 \times 10^{-19}$ & $6.40 \times 10^{-19}$ & -1.68 & 6.82 & $8.96 \times 10^{9}$ \\
\hline UGC 2885 & $1.17 \times 10^{11}$ & 0.846 & $1.53 \times 10^{-21}$ & 50 & $1.0 \times 10^{-22}$ & $2.80 \times 10^{-18}$ & $2.80 \times 10^{-18}$ & -1.5 & 74.7 & $3.00 \times 10^{11}$ \\
\hline \multicolumn{11}{|c|}{ Spirals with Desirable Characteristics } \\
\hline 1560 & $2.80 \times 10^{9}$ & 0.279 & $1.40 \times 10^{-21}$ & 6 & $4.0 \times 10^{-22}$ & $9.90 \times 10^{-20}$ & $2.60 \times 10^{-19}$ & -1.0 & 8.6 & $5.40 \times 10^{9}$ \\
\hline 2403 & $2.90 \times 10^{10}$ & 0.166 & $5.20 \times 10^{-22}$ & 17 & $1.1 \times 10^{-22}$ & $3.20 \times 10^{-18}$ & $9.20 \times 10^{-18}$ & -1.74 & 19.6 & $3.90 \times 10^{10}$ \\
\hline 2841 & $1.70 \times 10^{11}$ & 0.437 & $8.02 \times 10^{-22}$ & 12 & $5.0 \times 10^{-23}$ & $4.00 \times 10^{-17}$ & $2.85 \times 10^{-17}$ & -2.03 & 74 & $5.90 \times 10^{11}$ \\
\hline 2903 & $6.70 \times 10^{10}$ & 0.321 & $6.30 \times 10^{-22}$ & 28 & $5.0 \times 10^{-25}$ & $1.10 \times 10^{-17}$ & $1.10 \times 10^{-17}$ & -2.12 & 30 & $1.17 \times 10^{11}$ \\
\hline 3109 & $2.99 \times 10^{8}$ & 0.839 & $8.00 \times 10^{-22}$ & 5.6 & $7.0 \times 10^{-22}$ & $5.00 \times 10^{-20}$ & $1.80 \times 10^{-20}$ & -0.86 & 6.62 & $3.08 \times 10^{9}$ \\
\hline 3198 & $6.00 \times 10^{10}$ & 0.213 & $3.26 \times 10^{-22}$ & 43 & $1.4 \times 10^{-23}$ & $5.50 \times 10^{-18}$ & $3.90 \times 10^{-18}$ & -1.85 & 46.4 & $1.19 \times 10^{11}$ \\
\hline $4789 a$ & $1.88 \times 10^{8}$ & 0.234 & $3.90 \times 10^{-21}$ & 4.8 & $3.0 \times 10^{-23}$ & $2.00 \times 10^{-19}$ & $5.00 \times 10^{-20}$ & $-1.41^{2}$ & 7.40 & $1.97 \times 10^{9}$ \\
\hline 5585 & $5.90 \times 10^{9}$ & 0.290 & $1.01 \times 10^{-21}$ & - & $6.0 \times 10^{-22}$ & $4.12 \times 10^{-19}$ & $5.50 \times 10^{-19}$ & -1.39 & 10 & $9.00 \times 10^{9}$ \\
\hline 6503 & $9.58 \times 10^{9}$ & 0.253 & $1.84 \times 10^{-21}$ & 17 & $1.2 \times 10^{-22}$ & $1.10 \times 10^{-17}$ & $2.20 \times 10^{-19}$ & -2.09 & 19 & $2.9 \times 10^{10}$ \\
\hline 7331 & $1.47 \times 10^{11}$ & 0.362 & $8.70 \times 10^{-22}$ & 30 & $2.5 \times 10^{-23}$ & $9.00 \times 10^{-18}$ & $1.70 \times 10^{-17}$ & -1.89 & 31.6 & $1.98 \times 10^{11}$ \\
\hline
\end{tabular}


Table 5. Cont

\begin{tabular}{|c|c|c|c|c|c|c|c|c|c|c|}
\hline \multicolumn{11}{|c|}{ Visible Edge Properties } \\
\hline Name & $\begin{array}{c}M_{\text {in }} \\
\left(M_{\text {Sun }}\right)\end{array}$ & $\begin{array}{c}L_{\mathrm{vis}} / M_{\text {in }} \\
\left(L_{\text {Sun }} / M_{\text {Sun }}\right)\end{array}$ & $\begin{array}{c}\text { Density } \\
\left(\mathrm{kg} \mathrm{m}^{-3}\right)\end{array}$ & $\begin{array}{c}r_{\text {turndown }} \\
(\text { kpc) }\end{array}$ & $\begin{array}{l}\text { Lowest } \rho \\
\left(\mathrm{kg} \mathrm{m}^{-3}\right)\end{array}$ & $\begin{array}{c}\rho \text { at } 0.1 \mathrm{kpc} \\
\left(\mathrm{kg} \mathrm{m}^{-3}\right)\end{array}$ & $\begin{array}{c}\text { Highest } \rho \\
\left(\mathrm{kg} \mathrm{m}^{-3}\right)\end{array}$ & Power ${ }^{1}$ & $\begin{array}{l}r_{\max } \\
(\mathrm{kpc})\end{array}$ & $\begin{array}{c}M_{\max } \\
\left(M_{\text {Sun }}\right)\end{array}$ \\
\hline \multicolumn{11}{|c|}{ Messier Spirals } \\
\hline 1068 & $5.00 \times 10^{10}$ & 0.588 & $3.00 \times 10^{-21}$ & - & $3.0 \times 10^{-20}$ & $1.22 \times 10^{-17}$ & $1.22 \times 10^{-17}$ & -1.53 & 5.09 & $4.29 \times 10^{10}$ \\
\hline 3031 & $5.60 \times 10^{10}$ & 0.364 & $4.50 \times 10^{-22}$ & - & $9.3 \times 10^{-24}$ & $6.70 \times 10^{-18}$ & $9.60 \times 10^{-18}$ & -2.0 & 23 & $7.00 \times 10^{10}$ \\
\hline 4192 & $9.00 \times 10^{10}$ & 0.228 & $1.20 \times 10^{-21}$ & $?$ & $6.0 \times 10^{-22}$ & $1.66 \times 10^{-17}$ & $2.70 \times 10^{-17}$ & -1.74 & 17.7 & $8.65 \times 10^{10}$ \\
\hline 4254 & $>2 \times 10^{10}$ & $<1$ & $\sim 5 \times 10^{-21}$ & - & $\sim 2 \times 10^{-21}$ & $3.00 \times 10^{-18}$ & $3.00 \times 10^{-17}$ & -1.4 & 1.9 & $4.50 \times 10^{9}$ \\
\hline 4258 & $1.10 \times 10^{11}$ & 0.170 & $2.50 \times 10^{-22}$ & 24 & $6.3 \times 10^{-23}$ & $2.38 \times 10^{-17}$ & $6.00 \times 10^{-16}$ & -2.09 & 33.4 & $1.33 \times 10^{11}$ \\
\hline 4303 & $3.68 \times 10^{10}$ & 0.497 & $6.81 \times 10^{-22}$ & - & $5.0 \times 10^{-22}$ & $2.44 \times 10^{-17}$ & $5.00 \times 10^{-17}$ & -1.96 & 18.6 & $5.00 \times 10^{10}$ \\
\hline 4321 & $1.68 \times 10^{11}$ & 0.265 & $8.80 \times 10^{-22}$ & 22 & $1.7 \times 10^{-23}$ & $3.57 \times 10^{-17}$ & $6.70 \times 10^{-17}$ & -2.00 & 23.3 & $1.80 \times 10^{11}$ \\
\hline 4501 & $2.00 \times 10^{11}$ & 0.325 & $4.80 \times 10^{-21}$ & - & $6.8 \times 10^{-21}$ & $1.36 \times 10^{-17}$ & $3.60 \times 10^{-17}$ & -1.46 & 8.32 & $9.54 \times 10^{10}$ \\
\hline 4548 & $3.80 \times 10^{10}$ & 0.732 & $3.20 \times 10^{-22}$ & - & $1.5 \times 10^{-21}$ & $2.40 \times 10^{-17}$ & $3.50 \times 10^{-17}$ & -2.57 & 8.39 & $3.33 \times 10^{10}$ \\
\hline 4569 & $9.00 \times 10^{10}$ & 0.214 & $2.20 \times 10^{-21}$ & - & $3.7 \times 10^{-21}$ & $3.78 \times 10^{-17}$ & $6.39 \times 10^{-17}$ & -1.55 & 11.8 & $8.27 \times 10^{10}$ \\
\hline 4736 & $1.77 \times 10^{10}$ & 0.556 & $1.40 \times 10^{-21}$ & 6.3 & $1.7 \times 10^{-22}$ & $2.90 \times 10^{-17}$ & $2.90 \times 10^{-17}$ & -2.12 & 6.55 & $2.14 \times 10^{10}$ \\
\hline 5055 & $7.07 \times 10^{10}$ & 0.245 & $5.21 \times 10^{-22}$ & 16.5 & $2.4 \times 10^{-23}$ & $1.25 \times 10^{-17}$ & $2.63 \times 10^{-17}$ & -2.19 & 40 & $1.36 \times 10^{11}$ \\
\hline 5194 & $4.00 \times 10^{10}$ & 0.535 & $1.00 \times 10^{-22}$ & 6.5 & $1.0 \times 10^{-22}$ & $3.00 \times 10^{-17}$ & $7.00 \times 10^{-17}$ & -2.1 & 6.51 & $4.32 \times 10^{10}$ \\
\hline 5236 & $4.79 \times 10^{10}$ & 0.668 & $1.66 \times 10^{-22}$ & 19 & $1.0 \times 10^{-23}$ & $2.85 \times 10^{-17}$ & $4.48 \times 10^{-16}$ & -2.42 & 19.90 & $4.83 \times 10^{10}$ \\
\hline 5457 & $5.26 \times 10^{10}$ & 0.422 & $2.50 \times 10^{-22}$ & 9 & $1.7 \times 10^{-23}$ & $1.00 \times 10^{-17}$ & $1.50 \times 10^{-17}$ & -2.10 & 12.90 & $5.29 \times 10^{10}$ \\
\hline \multicolumn{11}{|c|}{ Dwarf Irregular and Spheroidal Galaxies } \\
\hline WLM & $8.63 \times 10^{7}$ & 0.676 & $9.20 \times 10^{-23}$ & 2 & $2.3 \times 10^{-23}$ & - & $6.60 \times 10^{-22}$ & $-1.9^{2}$ & 2.0 & $1.08 \times 10^{8}$ \\
\hline $\mathrm{M} 81 \mathrm{dWb}$ & $7.00 \times 10^{7}$ & 1.02 & $5.00 \times 10^{-22}$ & 0.8 & $4.5 \times 10^{-22}$ & $1.87 \times 10^{-19}$ & $1.90 \times 10^{-19}$ & $-2.2^{2}$ & 0.92 & $7.31 \times 10^{7}$ \\
\hline Holmberg II & $4.28 \times 10^{8}$ & 1.10 & $3.64 \times 10^{-22}$ & 7 & $1.6 \times 10^{-23}$ & $1.00 \times 10^{-19}$ & $2.20 \times 10^{-20}$ & -1.52 & 10.2 & $1.02 \times 10^{9}$ \\
\hline Carina & $3.38 \times 10^{6}$ & 0.087 & $6.50 \times 10^{-22}$ & - & $1.4 \times 10^{-22}$ & $8.70 \times 10^{-21}$ & $3.00 \times 10^{-20}$ & $-1.59^{2}$ & 0.88 & $6.88 \times 10^{6}$ \\
\hline Draco & $3.40 \times 10^{6}$ & 0.087 & $7.40 \times 10^{-22}$ & 0.5 & $1.3 \times 10^{-22}$ & $3.00 \times 10^{-20}$ & $3.10 \times 10^{-20}$ & -1.58 & 1.78 & $4.10 \times 10^{7}$ \\
\hline Fornax & $1.24 \times 10^{7}$ & 1.047 & $3.73 \times 10^{-23}$ & 1.4 & $2.0 \times 10^{-23}$ & $1.10 \times 10^{-20}$ & $2.00 \times 10^{-20}$ & $-1.56^{2}$ & 1.62 & $1.37 \times 10^{7}$ \\
\hline Leo I & $7.74 \times 10^{6}$ & 0.647 & $1.36 \times 10^{-21}$ & 0.8 & $1.8 \times 10^{-22}$ & $4.40 \times 10^{-21}$ & $2.00 \times 10^{-20}$ & -0.85 & 0.95 & $1.26 \times 10^{7}$ \\
\hline
\end{tabular}


Table 5. Cont.

\begin{tabular}{|c|c|c|c|c|c|c|c|c|c|c|}
\hline \multicolumn{11}{|c|}{ Visible Edge Properties } \\
\hline Name & $\begin{array}{c}M_{\text {in }} \\
\left(M_{\text {Sun }}\right)\end{array}$ & $\begin{array}{c}L_{\mathrm{vis}} / M_{\mathrm{in}} \\
\left(L_{\mathrm{Sun}} / M_{\mathrm{Sun}}\right)\end{array}$ & $\begin{array}{c}\text { Density } \\
\left(\mathrm{kg} \mathrm{m}^{-3}\right)\end{array}$ & $\begin{array}{c}r_{\text {turndown }} \\
(\mathrm{kpc})\end{array}$ & $\begin{array}{l}\text { Lowest } \rho \\
\left(\mathrm{kg} \mathrm{m}^{-3}\right)\end{array}$ & $\begin{array}{c}\rho \text { at } 0.1 \mathrm{kpc} \\
\left(\mathrm{kg} \mathrm{m}^{-3}\right)\end{array}$ & $\begin{array}{c}\text { Highest } \rho \\
\left(\mathrm{kg} \mathrm{m}^{-3}\right)\end{array}$ & Power $^{1}$ & $\begin{array}{l}r_{\max } \\
(\mathbf{k p c})\end{array}$ & $\begin{array}{c}M_{\max } \\
\left(M_{\text {Sun }}\right)\end{array}$ \\
\hline \multicolumn{11}{|c|}{ Lenticular and Elliptical Galaxies } \\
\hline UGC 3993 & $1.78 \times 10^{11}$ & 0.068 & $3.10 \times 10^{-22}$ & $18 ?$ & $4.0 \times 10^{-23}$ & $1.00 \times 10^{-17}$ & $8.00 \times 10^{-19}$ & -2.07 & 54.6 & $5.40 \times 10^{11}$ \\
\hline 7286 & $5.90 \times 10^{9}$ & 0.415 & $4.60 \times 10^{-22}$ & $?$ & $6.0 \times 10^{-23}$ & $6.00 \times 10^{-19}$ & $7.00 \times 10^{-20}$ & -1.71 & 15.7 & $1.77 \times 10^{10}$ \\
\hline 2768 & $1.98 \times 10^{10}$ & 1.41 & $1.00 \times 10^{-21}$ & 10 & $4.0 \times 10^{-22}$ & $2.75 \times 10^{-20}$ & $2.75 \times 10^{-20}$ & -0.61 & 21.8 & $1.96 \times 10^{10}$ \\
\hline 3379 & $1.10 \times 10^{10}$ & 1.92 & $9.00 \times 10^{-23}$ & - & $9.0 \times 10^{-23}$ & $3.64 \times 10^{-18}$ & $1.10 \times 10^{-17}$ & -2.38 & 5.38 & $1.32 \times 10^{10}$ \\
\hline 2434 & $5.00 \times 10^{10}$ & 0.476 & $1.00 \times 10^{-22}$ & - & $5.0 \times 10^{-22}$ & $2.95 \times 10^{-18}$ & $2.95 \times 10^{-18}$ & -2.02 & 8.22 & $4.54 \times 10^{10}$ \\
\hline 221 & $2.33 \times 10^{7}$ & 11.20 & $1.00 \times 10^{-22}$ & - & $8.0 \times 10^{-22}$ & $6.10 \times 10^{-20}$ & $4.20 \times 10^{-15}$ & -2.8 & 0.75 & $2.30 \times 10^{7}$ \\
\hline 4431 & $3.00 \times 10^{9}$ & 0.33 & $1.30 \times 10^{-21}$ & 2.6 & $1.3 \times 10^{-21}$ & $3.00 \times 10^{-20}$ & $3.00 \times 10^{-20}$ & $-0.8^{2}$ & 3.68 & $2.88 \times 10^{9}$ \\
\hline \multicolumn{11}{|c|}{ Polar Ring or Disk Galaxies } \\
\hline $4650 \mathrm{~A}$ & $1.90 \times 10^{10}$ & 0.51 & $1.0 \times 10^{-23}$ & 18 & $1.0 \times 10^{-22}$ & $1.28 \times 10^{-19}$ & $5.00 \times 10^{-19}$ & -0.87 & 13.0 & $1.60 \times 10^{10}$ \\
\hline
\end{tabular}


Milky Way density (Figure 3b) was determined using extended RCs [58,59]. Calculated densities increase as c/a decreases, per Equation (13). Density follows a power law over most of the Milky Way. Density falls off rapidly from 18 to $30 \mathrm{kpc}$, consistent with the existence of an edge, although the termination of the galaxy is not particularly abrupt. Near the galactic center, density depends weakly on $r$ (discussed further below).

Our calculated densities (Table 5) are consistent with independent measures (Table 1). Near the MW galactic center, density is like that of molecular cloud cores, whereas mid-galaxy densities are like those of typical molecular clouds. In the Solar Neighborhood, our results match the sum of the distributed star density plus the ISM density (cf. Tables 1 and 5). Further out, the density grades to lower values. Out to $2000 \mathrm{kpc}, \rho$ remains significantly higher than that of the cosmologically inferred density (Table 1), which is consistent with the Milky Way being part of a local group of galaxies that constitutes a concentration of matter in the universe

The average density in the innermost region of the MW varies more gradually than in the outer zones (cf. Figure 4). A power-law fit presumes a singularity at $r=0$, but the actual trend at the smallest values of $r$ is flatter. The real trend is neither fit by an exponential nor any other simple function. Therefore, two extrapolations were made to estimate conditions near the center. Extrapolating the mass trend suggests 1 star of Solar mass per sphere of 1 a.u. radius. Alternatively, assuming a constant density of 100 times the highest density defined by the data provides 1 Solar mass per 200 a.u. radius. Averaging these two estimates provides a density for the galactic center similar to that of the Dispersed Solar System (Table 1).

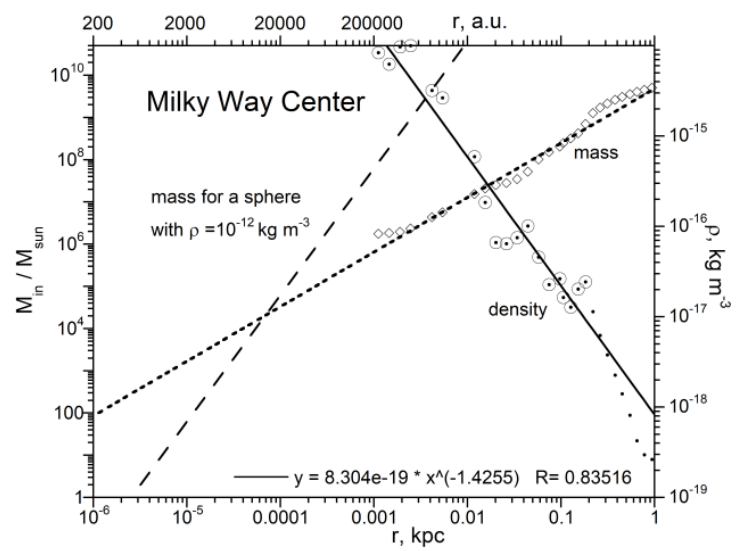

Figure 4. Expanded view of the Milky Way center. Mass and density are shown on left and right axes, respectively. The fit to $\rho$ is for $r<0.2 \mathrm{kpc}$. Central mass is estimated by extrapolating the mass curve (dotted line) and by assuming a high density (dashed line). See Figure 3 for data sources.

\subsection{Well-Studied and/or Illustrative Spiral Galaxies}

Compiled velocity for Andromeda [59] varies with $\mathrm{r}$ in an "oscillatory" pattern, similar to data for the Milky Way. Likewise, mass and density (Figure 3 from [27]; ) resemble values for the Milky Way (Table 5), but better conform to a power law. An edge was not obvious in either mass or density, even though the RC extends far beyond the visible edge at $23 \mathrm{kpc}$.

Three RC datasets for Triangulum are available $[14,65,66,70]$ and all agree (Figure 5), providing similar values for $\mathrm{M}_{\mathrm{in}}$. We used the smoother data sets to calculate densities. RCs barely cross the visible edge at $9.5 \mathrm{kpc}$, and thus do not define the density of its outer reaches. This galaxy is only half the size of MW and Andromeda yet has similar density from $r=1$ to $10 \mathrm{kpc}$. 

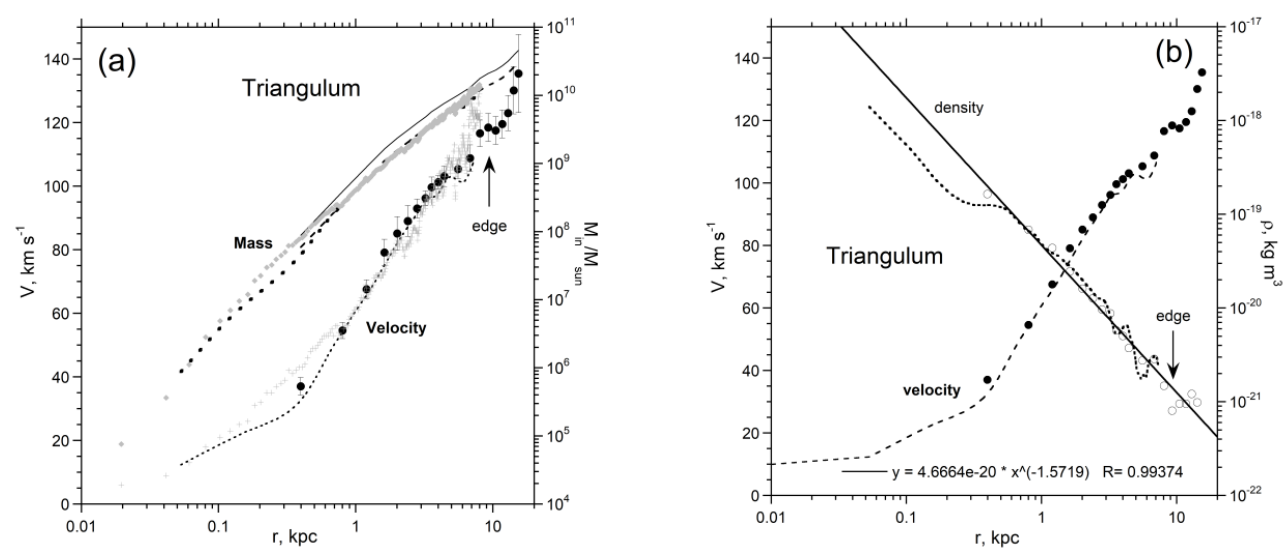

Figure 5. Comparison of three datasets on Triangulum to calculate mass (a) and density (b) vs. radius. RCs from Kam et al. [14] (grey crosses) were used to calculate mass (grey diamonds). RCs of Sofue [61] (black dashed line) were used to calculate mass (heavy black dotted line) and density (black dotted line). RCs of Corbelli and Salucci [70] (black points with error bars) were used to calculate mass for $c / a=1$ (solid line) and $c / a=0.1$ (medium dashed line) and density for $c / a=0.1$ (circles and solid line). Agreement is good and the masses extracted are similar. The fit to $\rho$ is over all $r$ of [70]. An abrupt drop off in density is not observed. RC data are limited to the visible disk.

The next few examples further probe how details in RCs affect calculations of mass and density. In the remaining figures, both mass and velocity are plotted against the left axis, which is linear, by scaling $\mathrm{M}_{\text {in }}$ by $10^{6}$ to $10^{9} \mathrm{M}_{\text {Sun }}$, as appropriate. Density is depicted by the logarithmic right axis, which facilitates comparison with independent measures (Table 1) and allows us to extrapolate to the central regions where velocity data are not available.

Sombrero (Figure 6) has an unusual shape. Jardel et al. [71] and Kormendy and Westphal [72] both indicate internal structure near $0.5 \mathrm{kpc}$, but neither RC extends beyond the visual edge at $14.4 \mathrm{kpc}$. We use the more extended data set for density calculations. A decrease in density exists at $0.4 \mathrm{kpc}$, but given the uncertainties, $\rho$ follows a power law, despite the zig-zag pattern for $v(r)$.
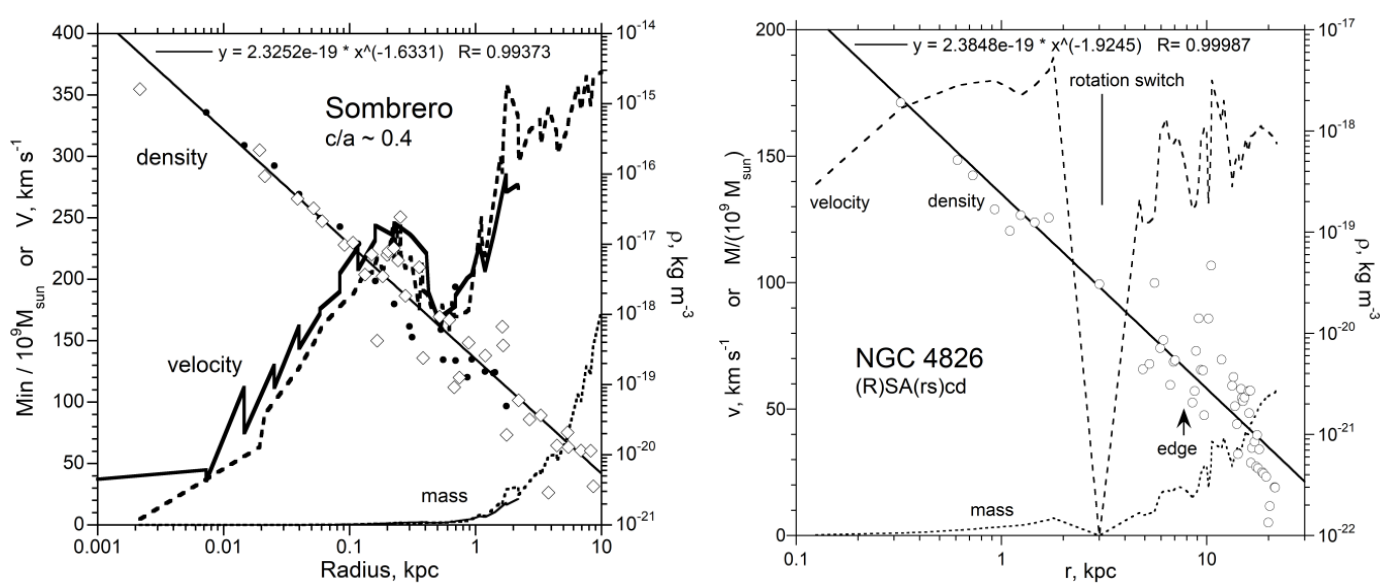

Figure 6. Inverse models of interesting spirals. Velocity and $M_{\mathrm{in}} /\left(10^{9} M_{\text {Sun }}\right)$ are both plotted on the left axis. (left) Sombrero galaxy, showing that disparities between RC measurements have little effect. Heavy solid line $=\mathrm{RC}$ of [71] was used to calculate $M_{\mathrm{in}}$ (light solid) and $\rho$ (open diamonds). Heavy broken lines $=\mathrm{RC}$ of [72] used to calculate mass (light broken line) and density (dots and fit). The visual edge is beyond the measurements; (right) Counter-rotating NGC-4826. RCs from deBlok et al. [54]. Results for mass and density are close to similar sized normally rotating NGC 7793 (Figure A3). We assumed that $v=0$ at $4 \mathrm{kpc}$, midway between the nearest counter-rotating data points. The breadth of the $v=0$ region affects the mass calculation, but little alters the density profile. 
NGC 2599 (Figure A2) has very high velocities near the center, but does not show the expected flat trend observed for many large spirals. Instead velocities decline further out. Nonetheless, the density profile is remarkably similar to that of Andromeda (Figure A1) for which the RC is oscillatory but is overall fairly flat.

Several other large galaxies have visual edge densities close to that of the ISM, whereas the calculated $\rho$ near galactic centers depends on the specific velocity profile. For example, studies of NGC 253 (Figure A2) have RCs that significantly differ at low $r[66,69]$. The calculated mass and density are affected at low $r$, but beyond a few kpc the differences are small. Hence, the total mass of the galaxy is indicated by the magnitude of $v$ at high $r$. The fits differ in detail, due to the contrasts in $v$ at low $r$, but the differences are not huge. The large spiral NGC 925 has low velocity (Figure A2) which indicates low density at its center. Moderate to strong differences in velocity for irregular NCG 3034 (Figure A3) give similar calculated masses. The differences in $v$ are more apparent in the densities; however, the effect is not large. Therefore, when asymmetries exist, averaging for more symmetric rotation curves should provide well-constrained mass and density.

Large UGC 2855 has an extended RC [62,66]. A drop off in density exists at high r (Figure A2). The power-law fit is good over an extended region, but density is flatter at small $r$ and steeper at large r. Densities are similar to those of the Milky Way and Andromeda.

Moderately large NGC 7793 (Figure A3) has lower central density (Table 5) than the aforementioned large galaxies. The pattern resembles that of the similar sized Evil Eye (Figure 6). Counter-rotation does not strongly affect the calculated density. Because the Virial theorem involves energies, the direction of rotation is not important to our calculations.

\section{3. "Representative" Galaxies}

Large galaxies (visual edge $>15 \mathrm{kpc}$ ) among the set examined by Bottema and Pestaña [64] (Figure A4) have similar mass and density profiles. Significantly smaller galaxies have much lower central density (Figure A5) like Triangulum (Figure 5). Otherwise, the profiles are similar to those of very large to moderately sized spirals. Even smaller dwarf DDO-154 has very low $\rho$ at the center (Figure 7a).
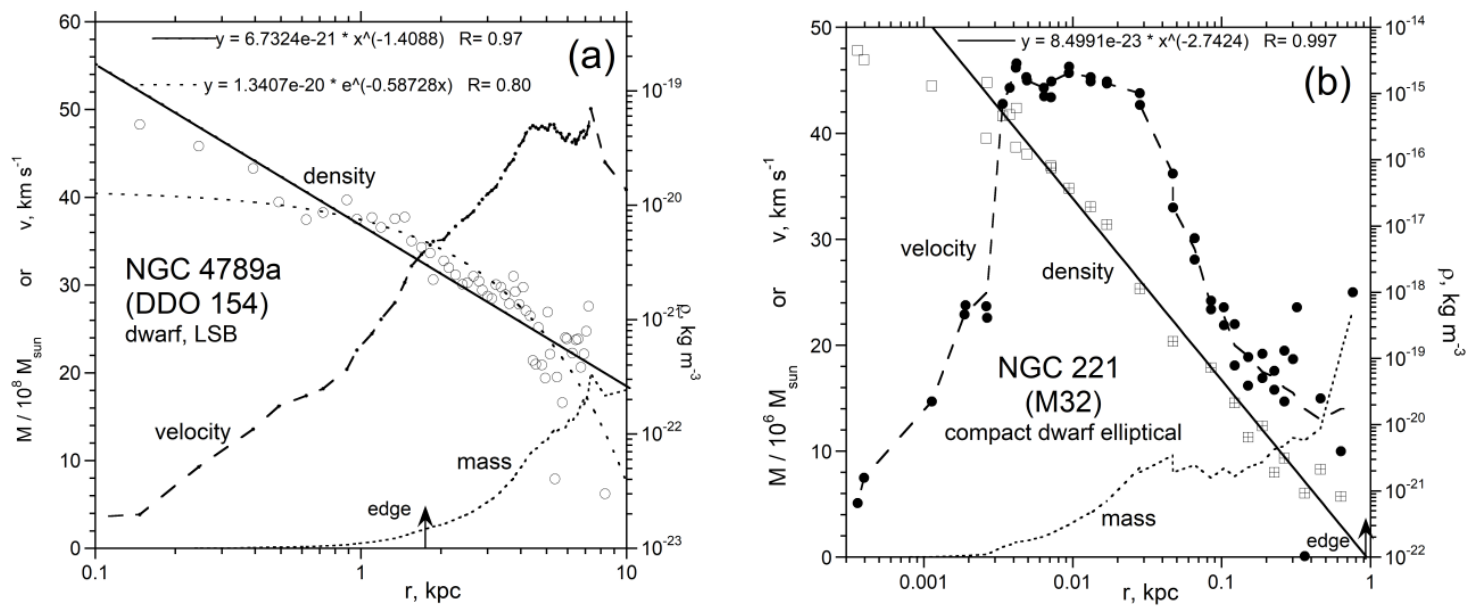

Figure 7. Small galaxies: (a) Low surface brightness, dwarf irregular galaxy NGC 4789a. RCs from Bottema and Pestaña [64]. $M_{\text {in }}$ ratioed to $10^{6} M_{\text {Sun }}$ Two fits to density are shown: solid = power law and dots = exponential; (b) Compact dwarf elliptical M32. RCs from Howley et al. [55]. $M_{\text {in }}$ ratioed to $10^{6} \mathrm{M}_{\text {Sun }}$. Density above $0.6 \mathrm{kpc}$ is described by a power law, but not an exponential.

\subsection{Messier Galaxies, including the Virgo Cluster}

The Messier examples are strongly skewed to large galaxies. Despite the variety of RCs $[53,61]$, calculated mass and density are quite similar (Figure A6; Table 5). 


\subsection{Dwarf Galaxies}

Rotation curves of the dwarfs Holmberg II, WLM, and M81dwb [73,74,85] (Figure A7) provide trends similar to those of DDO 154 (Figure 7a). For two of the four dwarfs, density decreases roughly exponentially with radius. The main difference between the dwarf irregulars and the largest spirals is the centrally concentrated density of the latter.

Dwarf spheroidals are smaller than irregulars and their even lower velocities [75-78] lead to lower mass and density (Figure A8). Central densities are very low (Table 5).

\subsection{Lenticular Galaxies}

RC of lenticular galaxies (e.g., [61]) resemble those of spiral galaxies, yielding similar mass and density profiles (Figure A9). As observed for the spirals, central density is small for small galaxies. The disk of NGC 2769 has lower density than its bulge, which is consistent with depiction of the density structure in terms of shells (Figure 1b). This galaxy is nearly elliptical.

\subsection{Elliptical Galaxies}

Elliptical galaxies have ambiguous orientations, precluding clear definition of their rotation curves, and so astronomers present their raw data as velocity dispersions. It must be recognized that uncertainties are large compared to spirals, and differential rotation between shells may involve different axial orientations. For the large ellipticals, the results are similar to spirals of like size (Figure A10). Smaller ellipticals [79] show more variety. High $\rho$ calculated at the center of NGC 221 (Figure 7b) may or may not be typical and no other data exist for this compact type. Yet, measurements of $v$ for NGC 221 are available for very small radii and do set a constraint on its interior (Table 5).

\subsection{A Polar Ring Galaxy}

Accurate RC data are available for only one polar ring galaxy, NGC4650a. Recent data of Iodice et al. [80] were analyzed (Figure 8). At small $r$, RCs of receding and approaching limbs of both the central discoid and the orthogonal polar discoid are all identical within uncertainty. A smooth variation of velocity across the entire object is seen, showing a common control. Velocities differ at large $r$ between the limbs, as has been observed in some other spirals. Because data from the receding polar limb are much less certain, we do not use this in our extraction. However, the fit for all data is not much different than that excluding the receding polar limb (Figure 8a). The data are well described by $v=\mathrm{A} r^{3}-\mathrm{B} r$ and resemble trends for many spirals (see Appendix A).
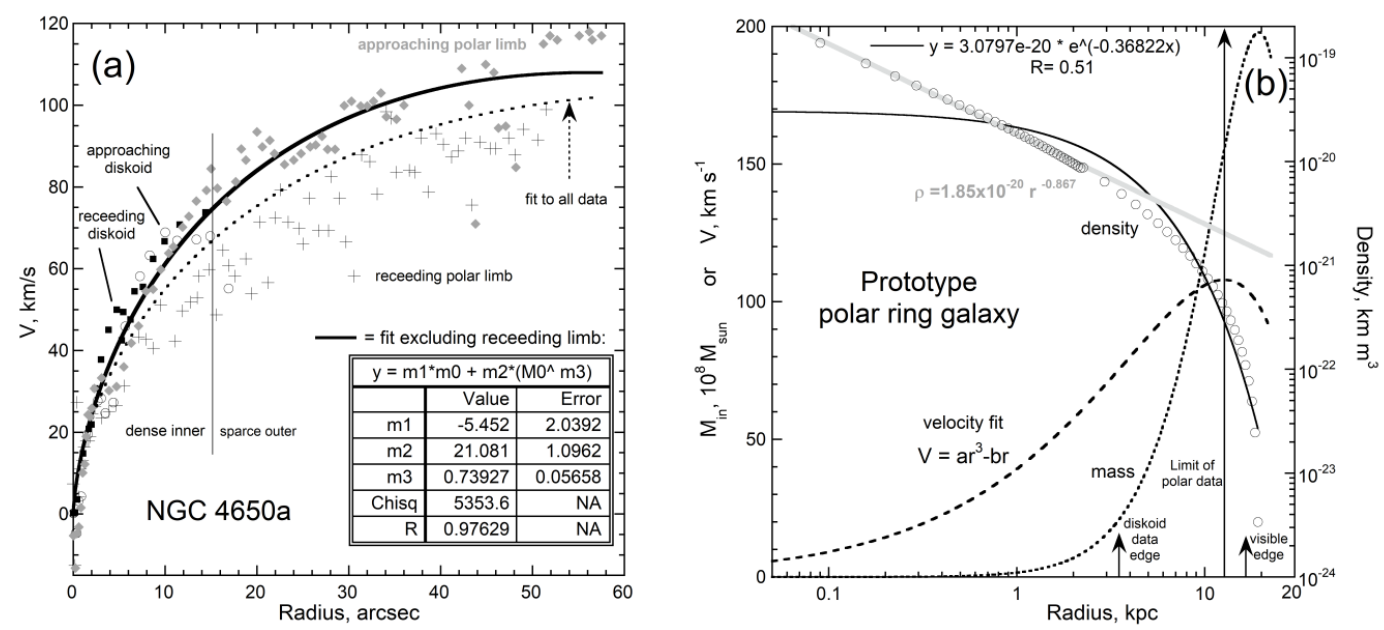

Figure 8. The prototype polar ring galaxy NGC 4650a. RC raw data from Iodice et al. [80] are similar to previous reports. (a) Comparison of RC data from different sectors and fits; see text. (b) The fit that excluded the uncertain dataset to provide $M_{\text {in }} /\left(10^{8} M_{\text {Sun }}\right)$ and $\rho$. 
The consistent, gradual change in $v$ implies gradual changes in $M_{\text {in }}$ and $\rho$ with $r$. Trends in extracted mass and density (Figure $8 \mathrm{~b}$ ) are similar to other spirals but less dense, more like the small galaxies (Figure 7). Density for NGC 4650a over all $r$ does not conform to an exponential function or a power law, but a definite outwards decline is seen, which is more pronounced towards the edge. Galactic dynamics of this polar ring galaxy differ little from that of spirals of similar size, other than the tilt of the inner discoid. Tilt angles are high, $\sim 90^{\circ}$ (NGCs 4650a and 4282) or $\sim 73^{\circ}$ (SPRC-7) [86].

\section{Discussion}

Profiles of density and mass vs. radius can be uniquely extracted from RC. These profiles are similar for all the galaxies examined, despite the wide range in shapes, sizes, and types (Table 4 ) and the variety of RC patterns exhibited (Figures 3-8, Figures A1-A10). Density tends to fall off as a power law, whereas values of $M_{\text {in }}$ rise strongly with radius. If velocity data are available near the galactic center (e.g., the Milky Way), then the extractions show that density at low $r$ depends more weakly on $r$ than it does at high $r$. If velocity data are available at great distance, density at very high $r$ sometimes steeply declines with radius, defining a physical "edge." In some cases, the visual and physical edges coincide (cf. Tables 4 and 5). Scatter existing in the calculated densities partly results from uncertainties in velocities, which can be substantial as suggested in Figure 3 and by comparing results of different studies of the same object (Figures 4-6). Despite the uncertainties, clear and consistent trends of extracted density with $r$ exist.

Our calculations (Figures 3-8, Figures A1-A10) show that the rotation curves are flat where the density dependence on radius approximately follows $r^{-1.8}$ (Table 5$)$. The following subsections compare our extracted $M_{\mathrm{in}}$ and $\rho$ with several measures that are entirely independent of RC determinations.

\subsection{Trends in Density with Galaxy Size and Morphology}

For all galaxies examined, $\rho$ at the visual edge does not vary much, such that the average value of $1.1 \times 10^{-21} \mathrm{~kg} \mathrm{~m}^{-3}$ matches ISM density (cf. Tables 1 and 5). The visual edge, being an isophote, is defined by a certain concentration of luminous matter in the galaxy. Association of the visual edge with a certain value of density (Figure 9, Figure 10a) indicates that total mass correlates with star mass. For spirals, density at the visual edge decreases with galaxy size (Figure 9a), ostensibly because larger galaxies have greater attractive power, thus producing more gradational outer reaches. For non-spiral morphologies, the trend at the visual edge is rather flat (Figure $9 b$ ).

Density at the visual edge for different spiral morphologies (types SA, SAB and SB) follow similar trends (not shown) to those in Figure 9a. Spiral type is not important to RC patterns [47] and thus not to extracted parameters. Rings or bars seem not to have a strong effect on density. However, tilt of the interior does have an effect: the polar-ring galaxy is less dense than others of its size. Essentially, this shape has more volume than the ordinary spirals, which are closer to being 2D objects than the obviously 3D ring type with mass well out of the equatorial plane.

The increase in density in the interior (at $0.1 \mathrm{kpc}$ ) with galaxy size is consistent with gravitation. For some small galaxies, the density may depend exponentially on radius (Figures 8b, A3, A5, A7, A8 and A9). However, exponential trends neither fit all small galaxies, nor any of our large galaxies (Figure A6). 

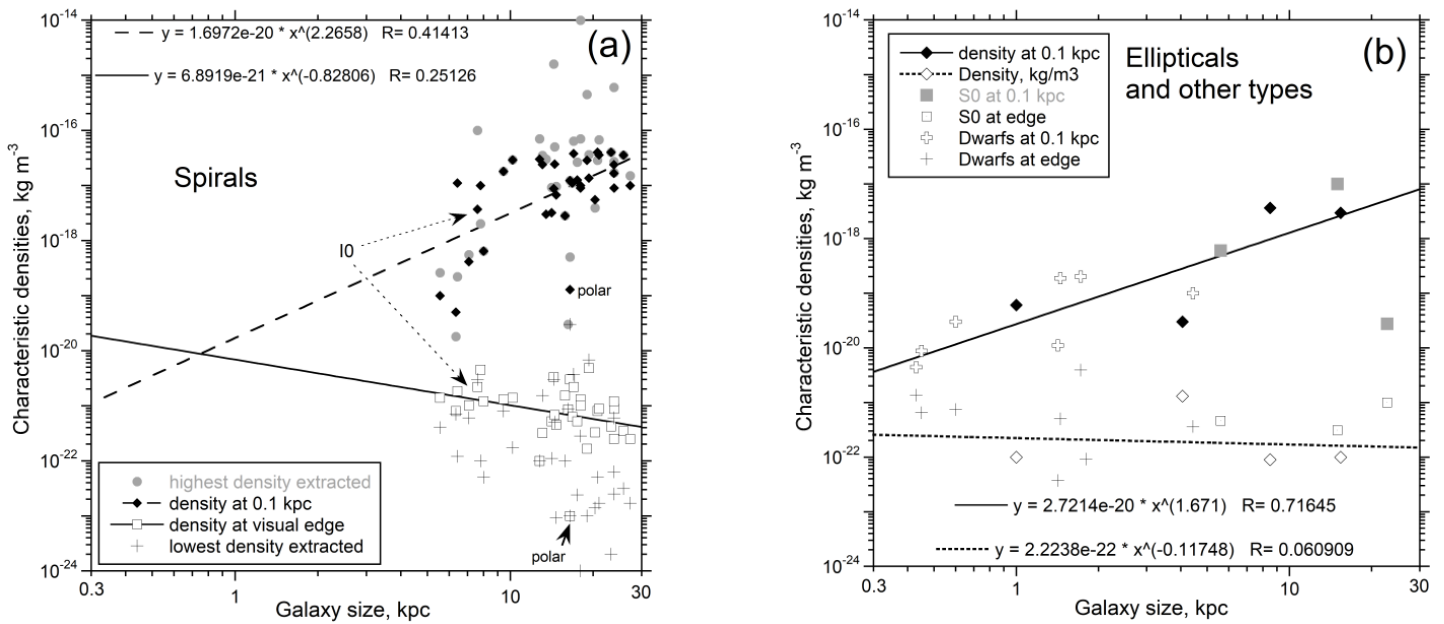

Figure 9. Various measures for the density of galaxies compared to their size, set to the visual edge at 25 B-magnitude $\operatorname{arcsec}^{-2}$. (a) Spiral and similar galaxies. M82 is included since near-IR images [23] suggest it is a spiral. Highest and lowest densities are depicted, but these values depend somewhat on smallest and largest radii explored in each RC study. For reference, we include $\rho$ measured at $r=0.1 \mathrm{kpc}$ and $\rho$ measured at the visual edge. The polar ring galaxy has lower $\rho$ than ordinary spirals of similar size. (b) Morphologies other than spiral. Fits to ellipticals (lines) roughly also describe the lenticular and spheroidal classes and are similar to fits for spirals.
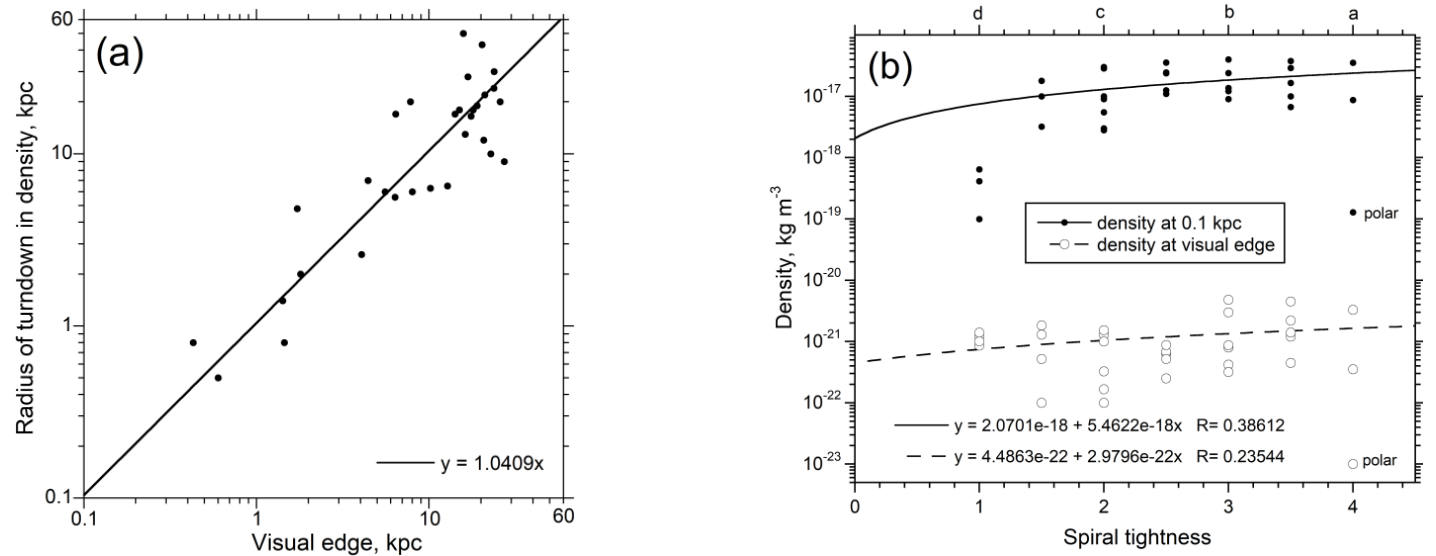

Figure 10. Density trends in spiral galaxies: (a) Radius of the turndown in density on the position of $0.1 \mathrm{kpc}$. Density at $0.1 \mathrm{kpc}$ strongly increases with the size of both spiral and elliptical galaxies (Figure 9a,b). The slopes of the regression lines for these types are similar, despite the disparity in the number of samples (36 spirals vs. 4 ellipticals), and disparities in central density between ellipticals and spirals. The three lenticular and eight dwarf galaxies measured also fall on or near these trends. The polar-ring type is less dense, due to its shape, with considerable matter out of the plane. Notably, the smallest galaxies, the dwarf spheroidals, have densities near the crossing of the trends and show little variation in $\rho$ with $r$. Interestingly, increasing tightness of the spiral arms is associated with higher $\rho$ at $0.1 \mathrm{kpc}$ (Figure 10b). This finding is consistent with spiral arms being concentrations of stars.

\subsection{Comparison of Extracted Density with Independently Known Densities}

Our calculations of galactic mass and density are supported by consistent trends and confirmed by independent measures of density (Tables 1 and 5). Near the centers of most galaxies, density is like that of molecular cloud cores, whereas at the middle of large galaxies, densities are like those of typical giant molecular clouds (GMCs). For some large galaxies, inner densities exceed those of GMC cores. Yet, even in this inner zone the calculated densities are lower than $\rho$ associated with evenly distributing the mass inside our Solar System (Table 1). 
At the radius of the Milky Way associated with our Sun, our calculated values for $\rho$ match the sum of the Solar Neighborhood density, calculated from the mass and distance of proximal stars, plus the ISM density (Tables 1 and 5). Further out in the Milky Way, the density grades to lower values, but even out to $\sim 1500 \mathrm{kpc}$, the radius of the local group, $\rho$ remains significantly higher than cosmological values. Our finding is consistent with the known existence of globular clusters, gas, and satellite galaxies surrounding the Milky Way, and with a large amount of material surrounding Andromeda, constituting $\sim 30 \%$ of total mass [87]. Furthermore, at $400 \mathrm{kpc}$ from centers of each of the Milky Way and Andromeda we obtain the same value for $\rho$ of $\sim 1.5 \times 10^{-24} \mathrm{~kg} \mathrm{~m}^{-3}$. This equivalence is consistent with separation of their centers by $780 \mathrm{kpc}$.

We next estimate IGM density by two approaches. The local group would have a density of $1 \times 10^{-26} \mathrm{~kg} \mathrm{~m}^{-3}$, if its total galaxy mass (Table 5) were uniformly redistributed out to a radius of 1.6 Mpc. Alternatively, the IGM for the local group can be determined by extrapolating the trends in Figures 3 and A1. RC data on the Milky Way projected to $1.6 \mathrm{Mpc}$ provide an upper limit of $1 \times 10^{-25} \mathrm{~kg} \mathrm{~m}^{-3}$. These two estimates are compatible with independent estimates of the IGM (Table 1).

When plotted against galaxy size, the trends for $\rho$ at $0.1 \mathrm{kpc}$ and at the visual edge diverge, suggesting an average density of $\sim 10^{-21} \mathrm{~kg} \mathrm{~m}^{-3}$ for all galaxies. The trends for the lowest and highest densities calculated are scattered, but their tendency to diverge (Figure 8a) also supports this "average" density. This crude average resembles that of the ISM and Solar Neighborhood (Table 1).

The highest central density, $10^{-14} \mathrm{~kg} \mathrm{~m}^{-3}$, was calculated for the Milky Way at $0.001 \mathrm{kpc}$ and for a compact dwarf ellipsoid at $0.0003 \mathrm{kpc}$ (Table 5). Yet, this value is far lower than the distributed $\rho$ of the Solar System (Table 1). The trend for the central Milky Way (Figure 4c) suggests that cores of large galaxies may reach 1 star per cubic a.u.

Rotation curves begin too far from galactic centers to precisely constrain how central $\rho$ depends on $r$. Exponential forms have been inferred from luminosity data (e.g., [88]). However, measured luminosity near the center is often more complicated than an exponential form and luminosity profiles generally end at $r$ below where RCs are collected. The densities we calculate for some small galaxies may have an exponential dependence. Velocities at small $r$ are needed to better quantify mass distribution near the center. Our calculations are consistent with ordinary matter being concentrated at galactic centers, but not inordinately so.

\subsection{Dependence of Galactic Mass on Size}

The calculated mass within the visual edge increases with galaxy size raised to the $\sim 2.7$ power (Figure 11a). A power of 3 is compatible with constant $\rho$, or with any trend where a common central density declines to a consistent value at the visual edge. Our result is consistent with a gradual increase in the average density of increasingly large galaxies. The dependence of $\rho$ on $r$ is weaker inside $0.1 \mathrm{kpc}$ than suggested by the power law fits extrapolated from values at larger $r$. Density may be nearly constant in the innermost zones of typical galaxies, where RCs cannot be determined.

The total mass of a galaxy is larger than $M_{\text {in }}$ at the visual edge, because the latter is an arbitrary cutoff, albeit a consistent one. The maximum mass extracted depends linearly on the radius corresponding to this mass (Figure 11b). However, the maximum mass could not be defined when the RC data were terminated close to the visual edge. Due to uncertainties in $v$ at large $r$, it is difficult to ascertain where the physical edge of a galaxy is located, if indeed such exists. Spiral galaxies grade into the gas of the IGM, whereas ellipticals and some dwarf galaxies have sharper edges (Figures 3-8; Figures A1-A10). These different behaviors are attributed to spirals having a much higher proportion of gas and dust (e.g., [89]). 

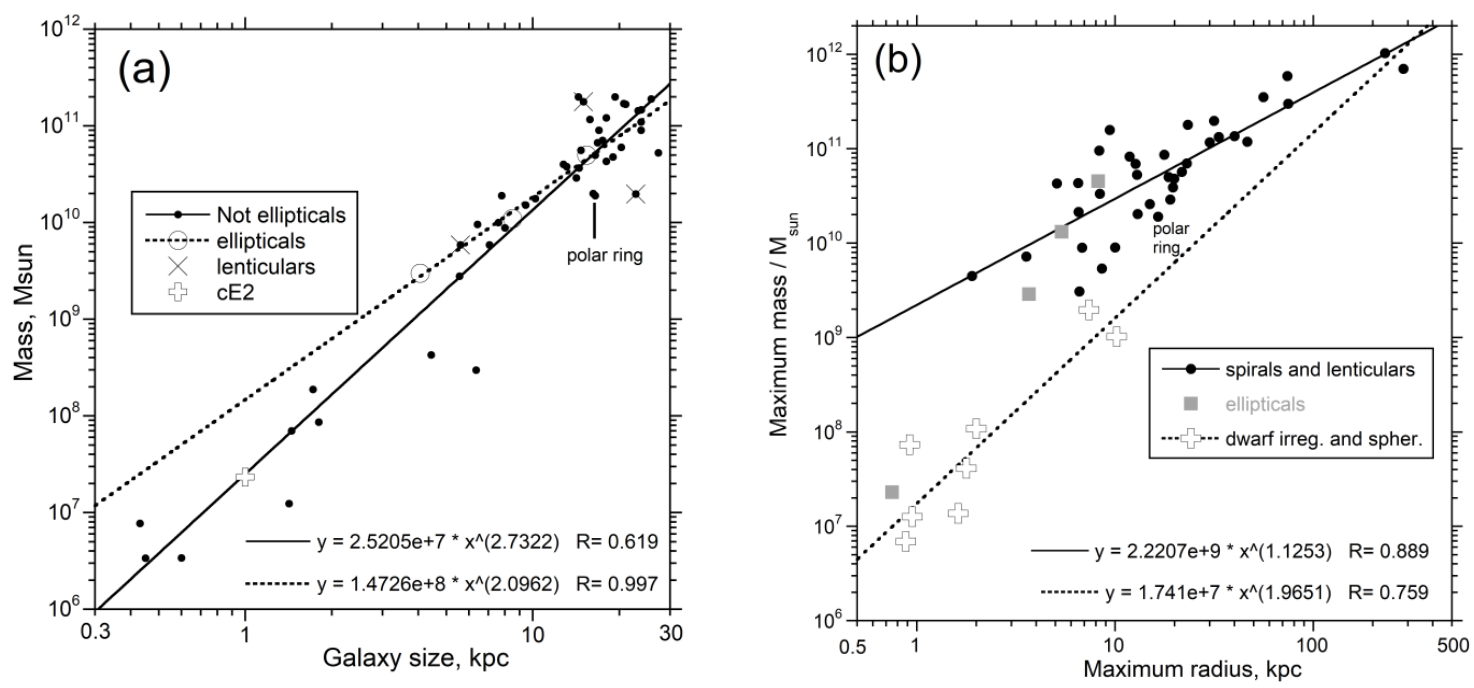

Figure 11. Galaxy mass-size relationships. (a) Dependence of galaxy mass on the position of the visible edge. (b) Dependence of the largest mass calculated for each galaxy on the radius where the largest mass was calculated. In most cases, this radius is the outer cutoff of available data, but some exceptions exist. Fits are to certain types, as listed in the inset.

\subsection{Relationships of Extracted Parameters with Luminosity}

Density depends on the visible luminosity in a manner that is similar to the dependence of density on size (cf. Figures 9 and 12). Density depends on radius to some power (Figures 3-8), whereby density is more concentrated to the centers of larger galaxies, which is consistent with known concentration of luminosity towards the center.
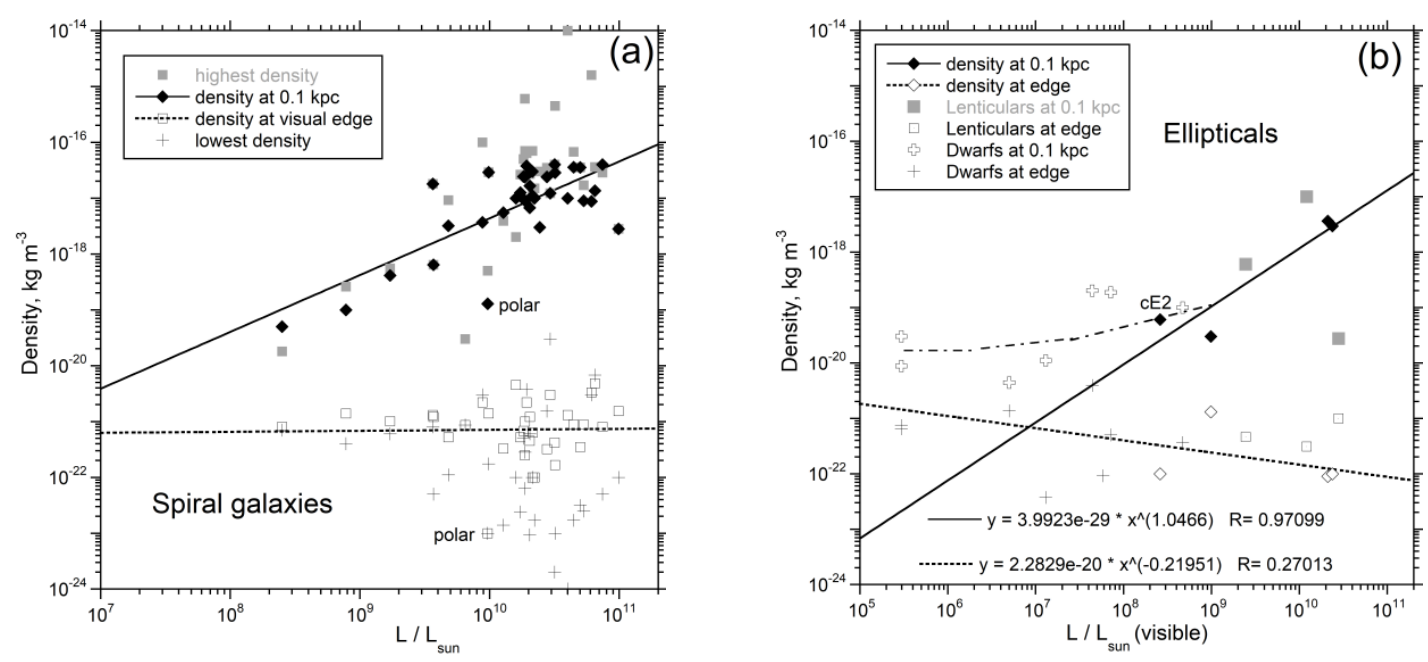

Figure 12. Dependence of density at various galactic positions on the visible luminosity of the galaxy: (a) Data for spirals and the polar-ring galaxy; (b) Other galaxy types, with a wider range of luminosity. The dwarf spheroidals show a flat dependence (dash-dotted curve). For the smallest galaxies, the average density is $\sim 10^{-21} \mathrm{~kg} \mathrm{~m}^{-3}$, i.e., where trends for the large spirals converge. 
Mass is proportional to the visible luminosity (Figure 13a). If we compare $M_{\text {in }}$ at the visual edge to measured $L_{\mathrm{vis}}$, then $L_{\mathrm{vis}} / M_{\text {edge }}$ is $\sim 0.4$ in Solar units for all types of galaxies, on average. This ratio is halved if the maximum mass is used (Figure 13a), which is consistent with most galaxy mass residing at high $r$ and young stars being at distance, because these require gas to form. For consistency, the measured luminosity in these figures are those reported in the NED [23]. Luminosity only measures surface brightness. Therefore, galactic mass should lie above the $M=L$ line in Figure 13 a, as observed for almost all galaxies.
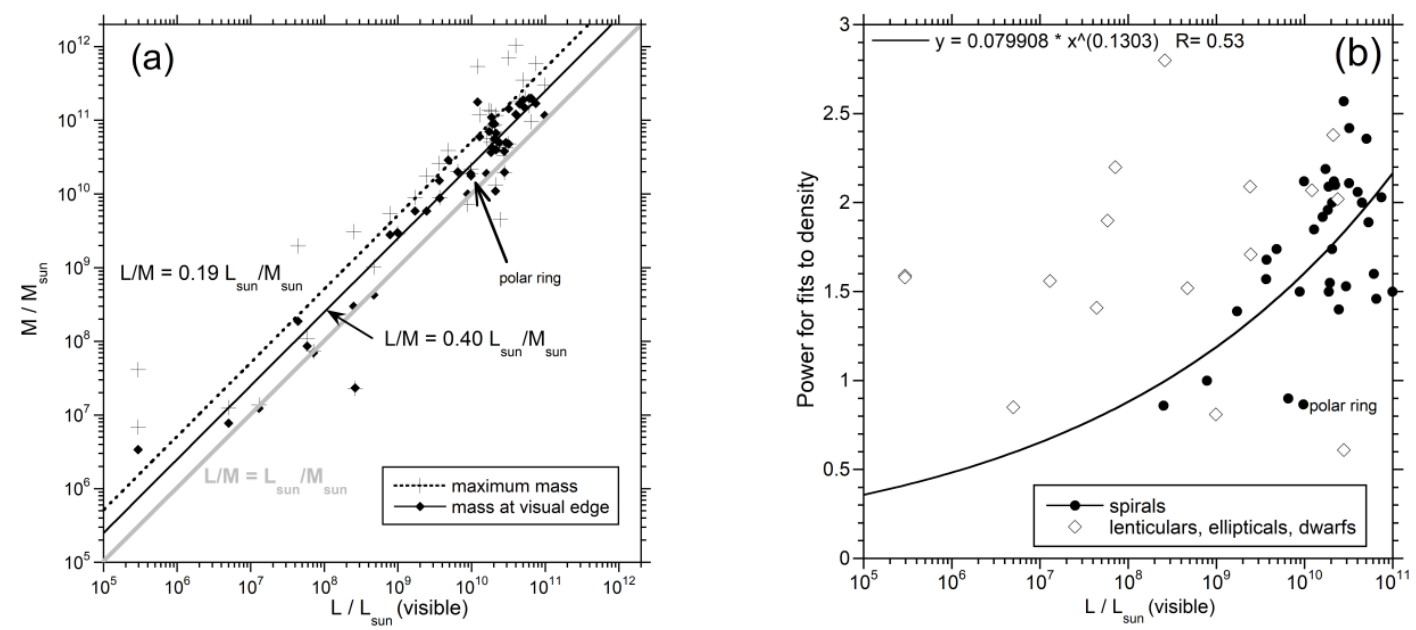

Figure 13. Dependence of calculated parameters on measured visible luminosity of our galaxy sample. Least squares fits are shown: (a) Mass of all galaxy types. The maximum mass is affected by the termination of velocity measurements with distance. The visual edge provides a more consistent measure of galaxy size in relationship to luminosity. Power law fits give exponents very close to unity: the linear fits shown are similar. Grey line indicates a 1:1 correspondence; (b) Power $(-n)$ for fits to density vs. radius. The least squares fit for spirals (solid curve) shows that density is more concentrated in the centers of larger galaxies. The polar-ring galaxy is less concentrated than normal spirals of similar size.

The relationship of luminosity to mass is affected by several additional factors. The distribution of star types is important, because luminosity for main sequence stars is $m_{\text {star }}{ }^{4}$ (e.g., [90]). A second factor is the number of white dwarfs and other dense stars which contribute significantly to mass but negligibly to luminosity: Ledrew's [32] independent assessment of the Solar Neighborhood provides $L_{\text {vis }} / M=0.8$, which reflects combined main sequence stars and white dwarfs. The third factor is presence of $\mathrm{H}$ atoms, gas, and dust, which will further reduce $L_{\mathrm{vis}} / M$ of a galaxy. The average $L_{\mathrm{vis}} / M$ $=0.4$ determined here is compatible with the Solar Neighborhood value of [32] because the later compares star mass to gas mass when scattering is unimportant to the measurement, and conversely for the former. Note that overall, the elliptical, lenticular, and dwarf galaxies have higher $L_{\mathrm{vis}} / M$ than the spirals, which is consistent with the spirals having considerably more gas (e.g., [89]). The fourth factor is size (Figure 14). Size has an effect because bigger galaxies are denser, as shown above, and thus pack more stars into a smaller volume. With this in mind, M32 owes its relatively high luminosity to being compact. 


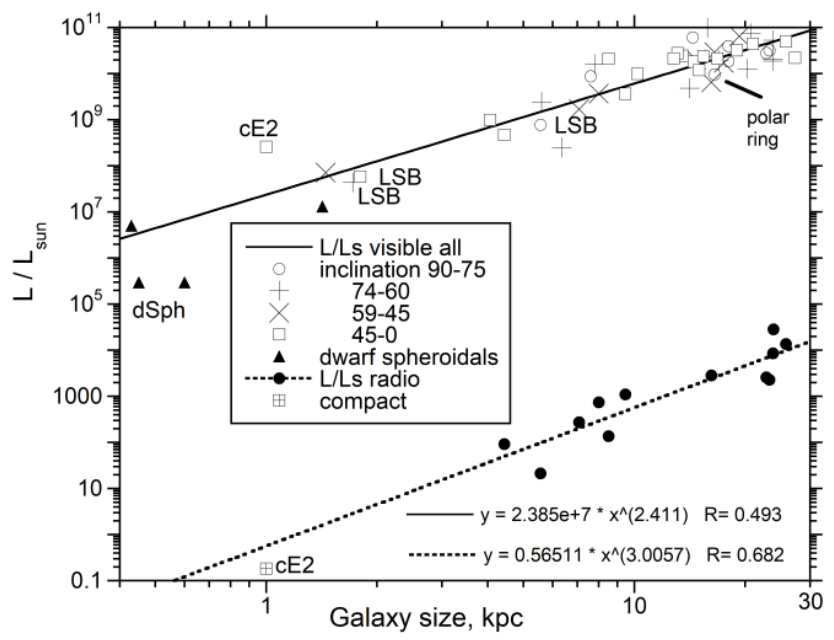

Figure 14. Dependence of luminosity in the visible and at $21 \mathrm{~cm}$ wavelengths on galaxy size, as determined from the visual edge at 25 B-magnitude $\operatorname{arcsec}^{-2}$. Data compiled in Table 4. Fits exclude the compact ellipsoid, M32.

\subsection{Non-Baryonic or Non-Luminous Matter?}

In addition to the above correlations, independent measurements of the visible luminosity of the 51 galaxies and of their visual size from the NED [23] are linearly correlated (Figure 14). This correlation neither depends on the angle of inclination nor on morphology, except that the compact ellipsoid (NGC 221) is far brighter for its size than the other 50 galaxies. Likewise, luminosity at the radio $(21 \mathrm{~cm})$ emission line of atomic $\mathrm{H}$ follows a parallel trend with the visual radius, again excepting NGC 221 (Table 5; Figure 14). Similar power laws suggest that the star mass and the H mass in a galaxy are correlated, which is consistent with luminous stars being mostly hydrogen.

The solid body approximation to spin provides mass at the visible edge that is consistent with luminosity $[5,6]$. Here, we deduce densities from detailed velocity data without this approximation and find that these densities are consistent with independent measures of baryonic matter. We have shown that flat RCs are expected for a rotating galaxy which becomes more dilute with extent. The flatness of the RC occurs where the decrease in density with $r$ offsets the growth in volume with $r$ : a large halo of dark matter is unnecessary.

The inner part of typical rotation curves features an increase in $v$ as $r$ increases. A linear increase in velocity indicates that density is nearly constant as $r$ increases in this innermost zone.

The mid-point of the Milky Way has $\sim 1$ gas mass to $\sim 4$ star masses, determined by comparing measured densities to those calculated at $8 \mathrm{kpc}$. If this ratio represents the entire Milky Way, then its $L / M$ should be about 2 , if the surface brightness includes all emitted light, which is clearly not the case. Importantly, gas increases to the outside of spirals, as does mass. Thus, $L / M$ of the whole galaxy must be reduced from that inferred from Solar Neighborhood properties and is compatible with the average $L / M=0.4$ at the edge determined here.

The connections found here are compatible with independent assessments of galactic structure by Disney et al. [91] who showed that one parameter governs galactic structure. This key parameter is baryonic mass, which is directly connected with galaxy size (Figure 11).

\section{Conclusions}

Evaluation of galactic rotation curves is greatly simplified by the combined use of Newton's homeoid theorems, Clausius's Virial theorem, and Maclaurin's gravitational self-potentials. These powerful theorems can be applied to galaxies which possess the expected spheroidal shape of a spinning self-gravitating body (Figure 1b). Existing orbital models include errors in mathematical physics [6] and cannot explain the rotational curves for galaxies based on detectable matter. 
Previous efforts are forward models of RC, which presume the cause, i.e., the density structure for each of multiple shapes is specified. Rotation curves are then calculated from the resulting multicomponent mass distributions (e.g., 12-14,41,54,55,64,68,70,74,86). In contrast, the present paper applies the inverse model of [27] for differentially spinning, variable density oblate spheroids to 51 different galaxies having detailed RC data. Our simple and exact equations for the homeoid allow density and mass distributions of individual galaxies to be directly and uniquely calculated from their measured RC and aspect ratio. Neither fitting parameters nor deconvolution are required. Groetsch [38] clarifies the differences between forward and inverse models and provides many examples of the latter.

Calculated densities are almost independent of galaxy type. Calculated densities in the inner parts of galaxies approximate those in GMC cores, whereas those closer to the middle zones approximate those of average GMCs (cf. Tables 1 and 5). Densities calculated from RCs for the Milky Way near the Sun's position are consistent with the independently determined distributed density of proximal stars. Slightly further out, extracted densities match ISM determinations, which is reasonable. In the outermost zones, density grades into, but is higher than, previous estimates of IGM values. We calculate IGM density for the local group as $>10^{-26} \mathrm{~kg} \mathrm{~m}^{-3}$.

Almost all galaxies are described by density depending on radius to a power $n$. The statistical average for the powers of the 36 individual spirals is $n=-1.80 \pm 0.40$. The 15 galaxies with other morphologies have similar average of $n=-1.63 \pm 0.63$. Including the polar ring galaxy in the spiral category negligibly affects these values.

Importantly, our results explain the empirically determined dependence of luminosity on velocity for each of the spiral and elliptical types (the Tully-Fisher and Faber-Jackson relationships). Our extracted masses are consistent with measured luminosity, given the presence of significant amounts of gas and some scattering. Our luminosity to mass ratios are consistent with the independent assessment of the Solar Neighborhood by Ledrew [32].

Our results for ellipticals and spirals differ only in detail, despite velocity dispersions being relevant in the first case and actual rotation curves in the other. Without friction, homeoids can rotate independently, as exemplified by counter-rotating spirals (e.g., NGC 4826) and polar-ring galaxies (e.g., NGC 4650a). For the latter case, the outer, less-dense region is tilted with respect to the inner, dense region, yet the RC curve is continuous, consistent with self-gravitation. Unlike flat spirals which must have co-axially spinning homeoids, in ellipticals, the rotation axes of homeoids can point in various directions, producing velocity dispersions.

We have shown that rotation curves of 51 galaxies covering diverse types and sizes can be explained by Newtonian physics without invoking non-baryonic matter.

Author Contributions: Both authors contributed more or less equally to all aspects and components of this research. All authors have read and agreed to the published version of the manuscript.

Funding: This research was partially funded by the National Aeronautics and Space Administration, grant number NNX13AB32A-3971105.

Acknowledgments: This research has made use of publicly available data: (1) the NASA/IPAC Extragalactic Database (NED), which is operated by the Jet Propulsion Laboratory, California Institute of Technology, under contract with the National Aeronautics and Space Administration and (2) data from the CHANG-ES project (Continuum Halos in Nearby Galaxies) involving the Karl G. Jansky Very Large Array.

Conflicts of Interest: The authors declare no conflict of interest. The funders had no role in the design of the study; in the collection, analyses, or interpretation of data; in the writing of the manuscript, or in the decision to publish the results.

\section{Appendix A}

Analyses of individual galaxies are organized after Table 4 and labeled by NCG number. Similar sized galaxies are grouped together. Except for Figure A1, velocity (dashed, sometimes with various symbols) and $M_{\mathrm{in}} /\left(X M_{\text {Sun }}\right)$ (dotted curve) are both plotted on the left axis, where the value of $X$ that was selected for scaling is indicated (e.g., $10^{6}$ to $10^{9}$ ). Density (various symbols) is plotted against the right axis. Power fit to density is shown as a solid line. Exponential fits are shown as widely 
spaced dotted lines. Results of fitting are listed. For size comparisons, we use the visual edge at 25 B-magnitude $\operatorname{arcsec}^{-2}$ provided by the NED [23] and illustrate this with an arrow.
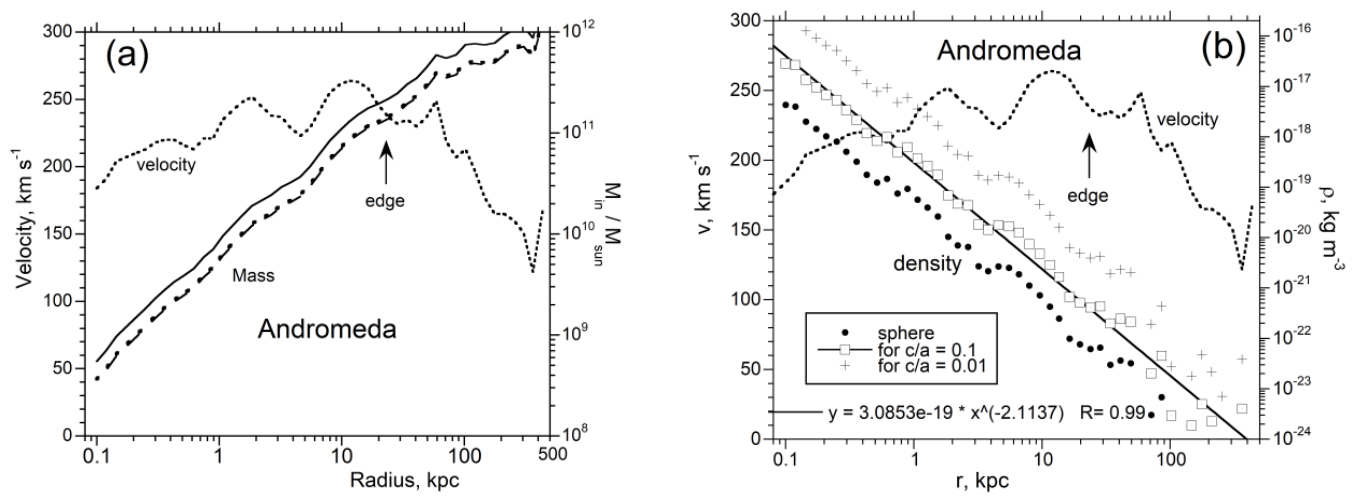

Figure A1. Andromeda. Inverse models showing the effect of varying $c / a$ for an externally probed galaxy, with the most extensive RCs available. Compiled RCs of Sofue [59] are used to calculate: (a) $M_{\text {in }}$ and; (b) density vs. radius. Labels on curves list the aspect ratios considered.
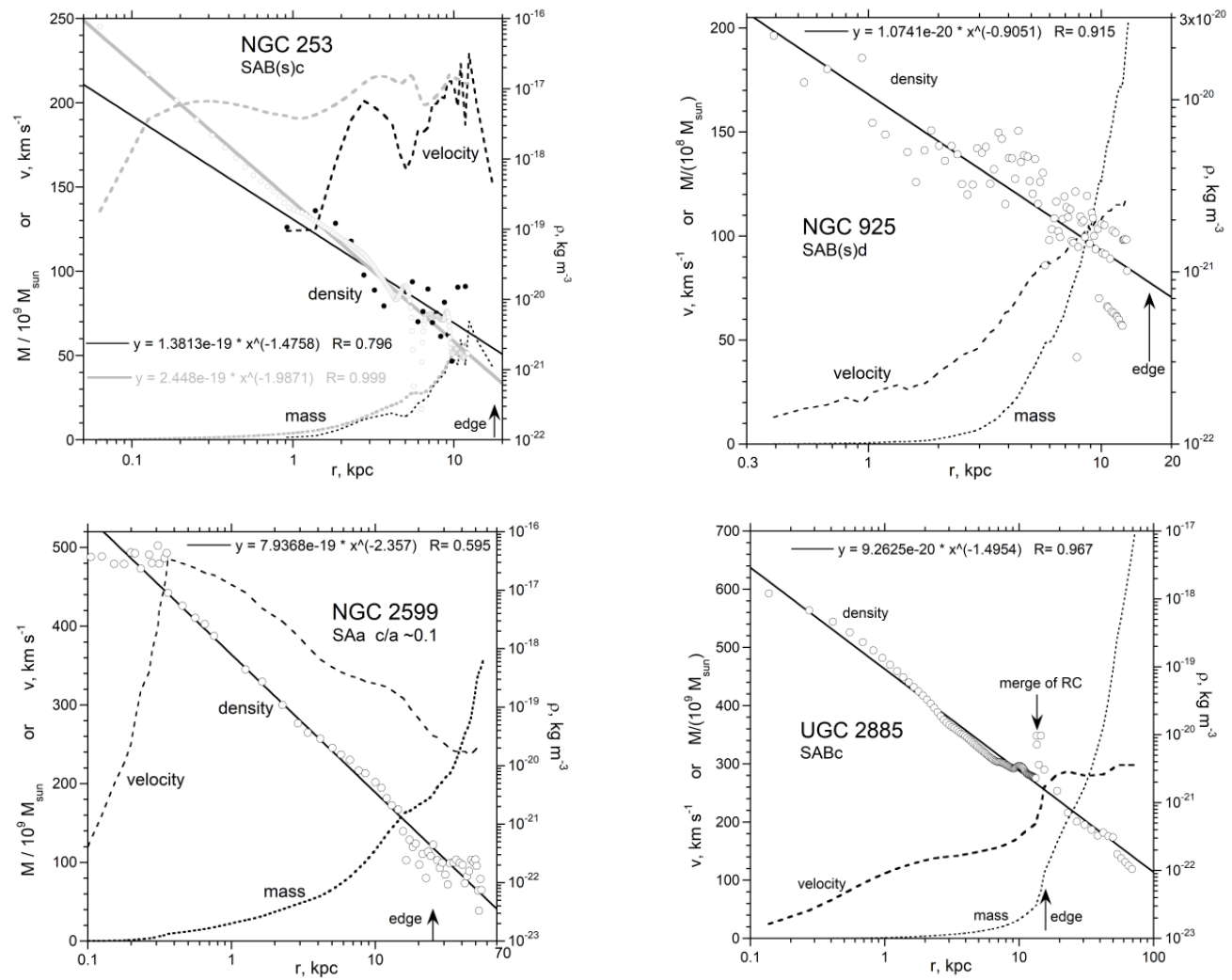

Figure A2. Large spirals: NGC 253 RCs from Sofue et al. [66] and derived $M$ and $\rho$ are colored grey. RCs from Hlavacek-Larrondo et al. [69] are black curves. Although velocity data differ substantially, similar mass and density are obtained near the visible edge. Differences at small $r$ affect the fits to $\rho$; NCG 925 has low velocities which produce low density; NGC 2599 RCs from Noordemeer et al. [61]. This spiral has both high $v$ and a strong decline in $v$ with radius. The increase in $v$ at low $r$ was assumed; UCG 2855 RCs below $13.7 \mathrm{kpc}$ from Sofue et al. [66], and RCs above $13.7 \mathrm{kpc}$ from Roelfsema and Allen [62]. The merge produces unrealistically high density over a short interval but does not seem to affect the fit. A drop off in density exists at high $r$, whereas the trend is flatter than the fit near the galaxy center. 

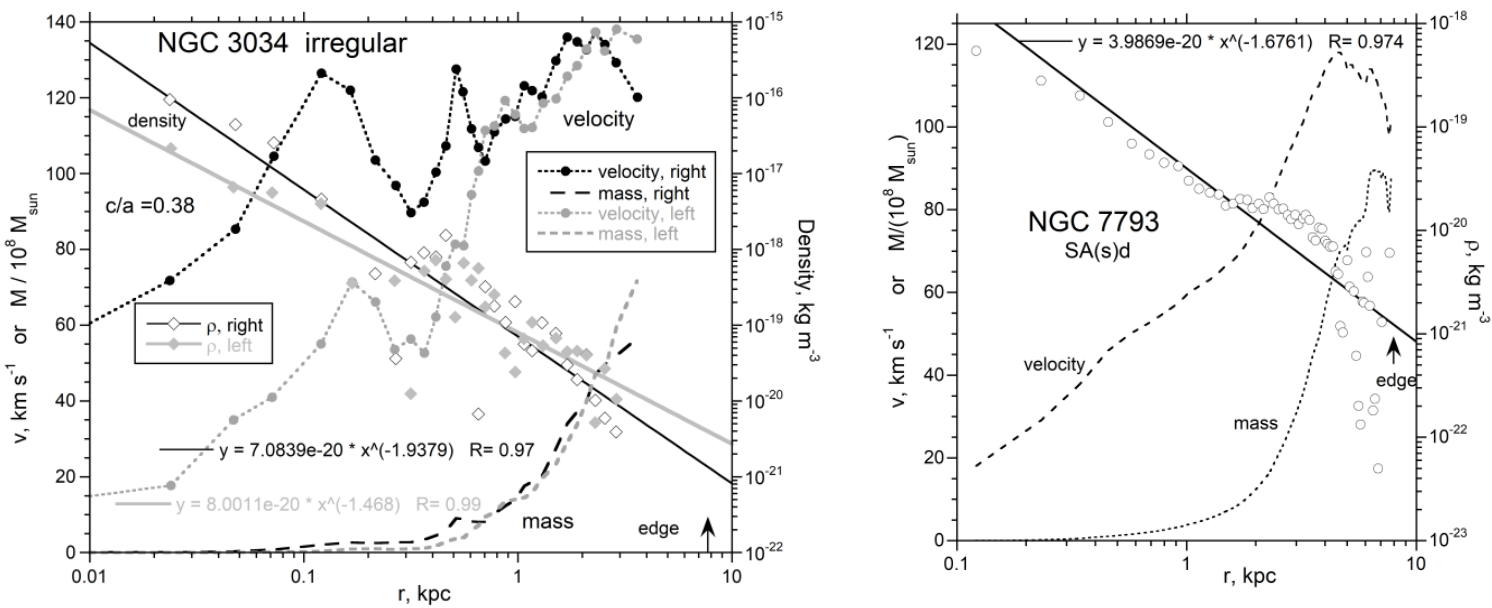

Figure A3. Comparison of moderate-sized galaxies: NGC 3034 is the irregular M82 galaxy. RCs from Greco et al. [63]. An abrupt drop off in density is not observed, because RC data are limited to the visible galaxy. NGC 7793 RCs from deBlok et al. [54] have fairly low velocity, and lower density.
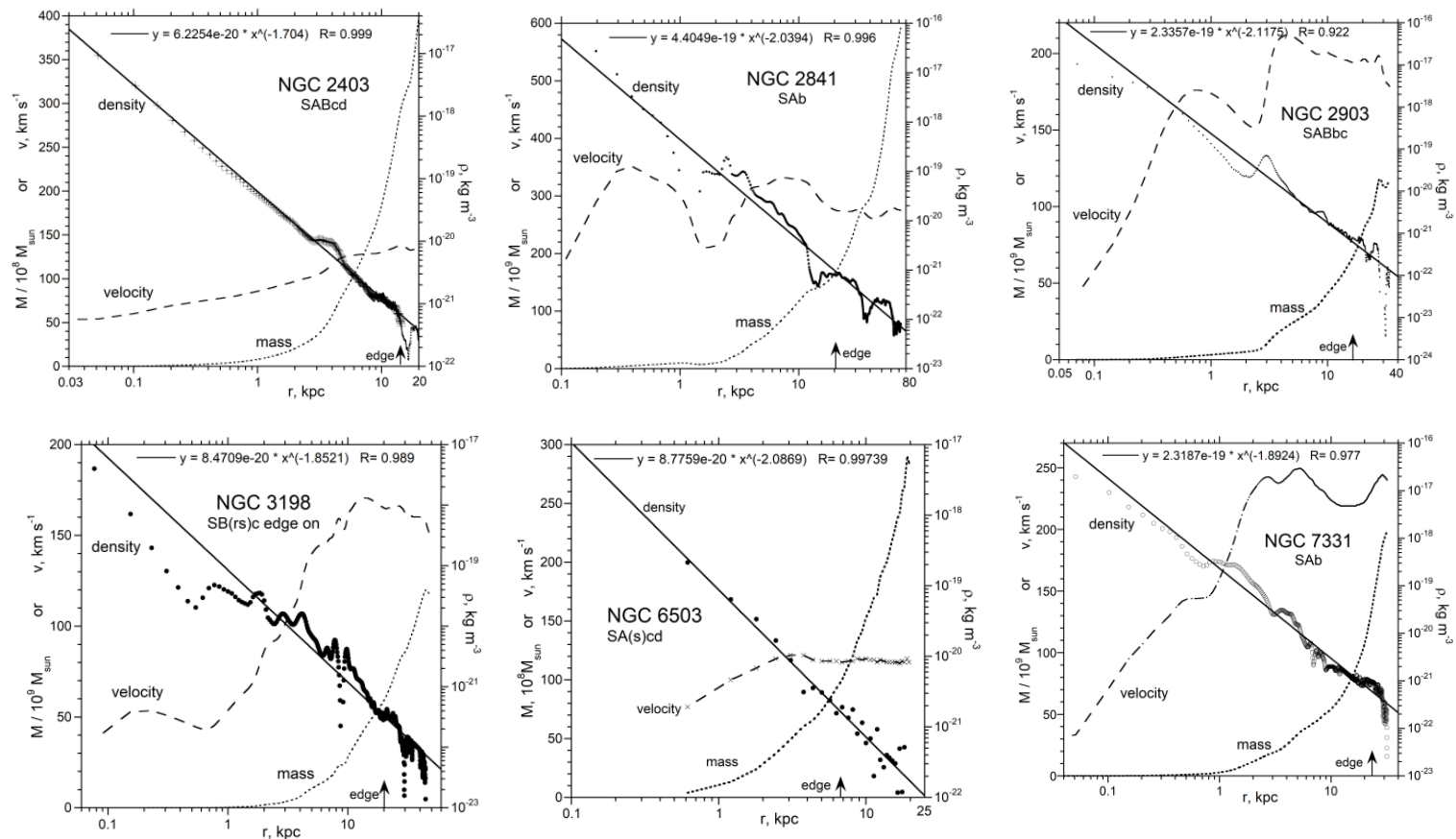

Figure A4. High-velocity large spirals from the compilation of Bottema and Pestaña [64]. Inner densities for similar visible edges depend on velocities.
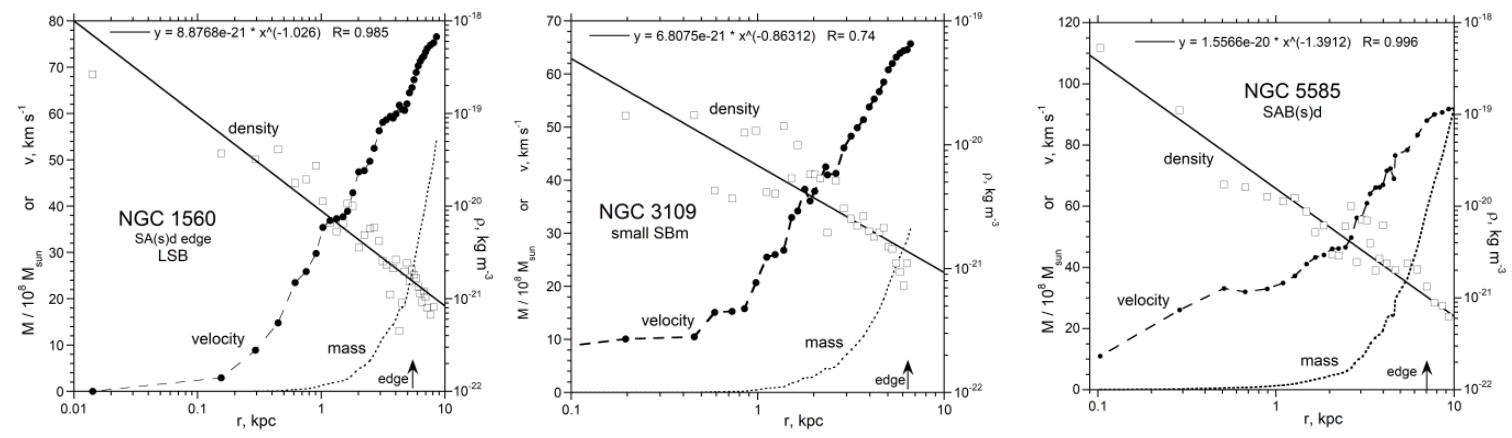

Figure A5. Three small spirals: RC data from Bottema and Pestaña [64]. 

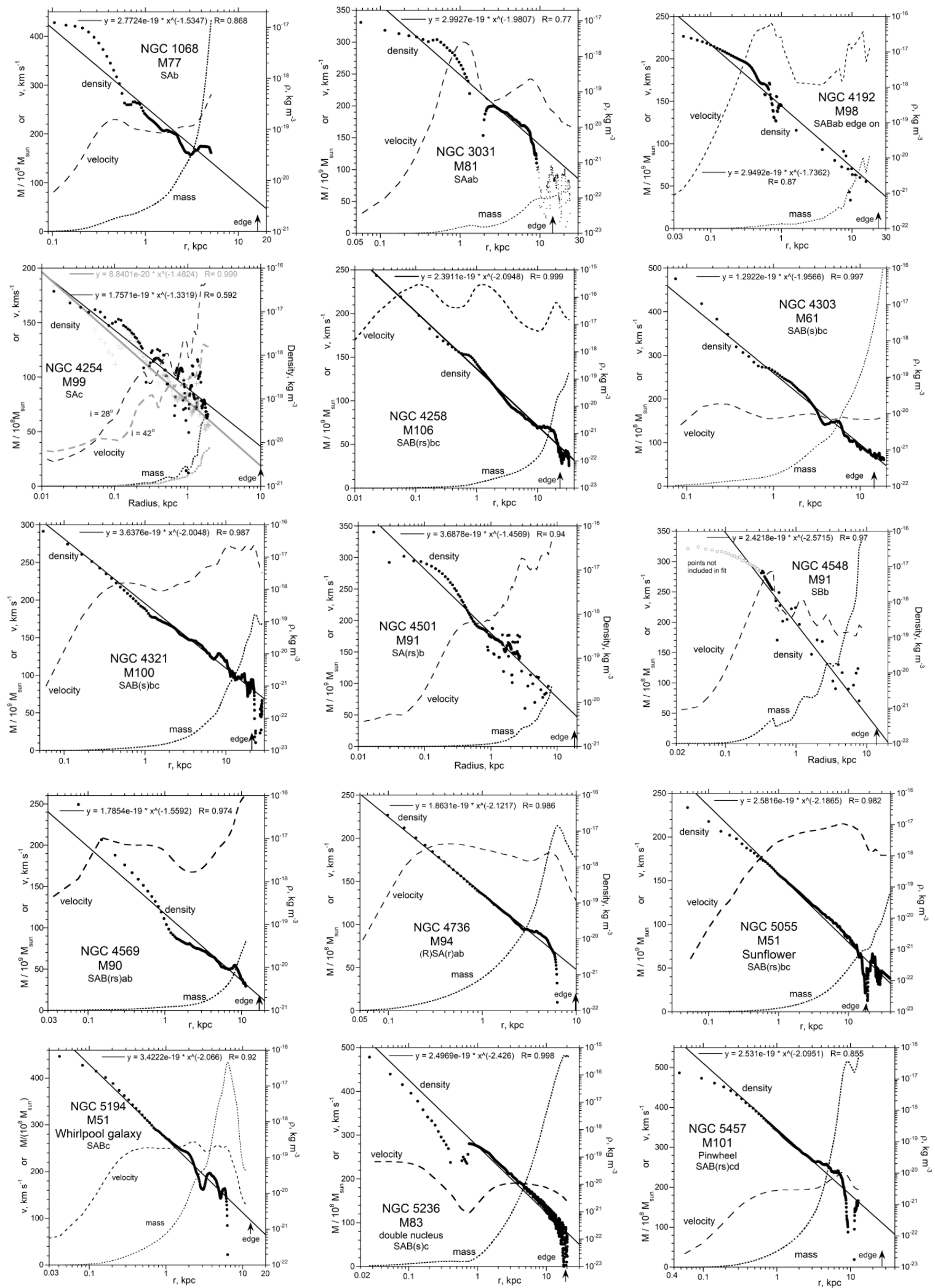

Figure A6. Spirals in Messier's Catalog studied by Sofue et al. [53,66]. These large and luminous galaxies have a variety of RC patterns. Tabular data were downloaded from [65]. 

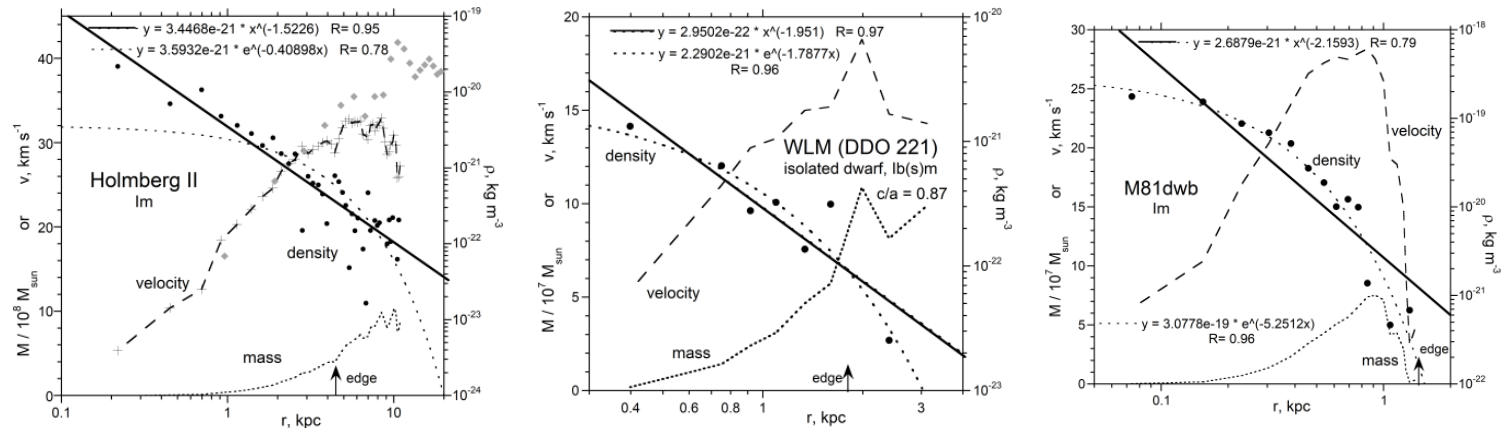

Figure A7. Irregular galaxies: For Holmberg II, squares and dashed line = RCs from Oh et al. [74]. Grey diamonds = RCs from Bureau and Carnigan [85], who state their velocities are not accurate beyond $10 \mathrm{kpc}$, and so this dataset was not used in the calculations. Dotted line $=$ calculated mass. Circles $=$ calculated density. Two different fits are shown as solid line and widely spaced dotted curve; WLM is unusual due to its isolation RCs from Leaman et al. [73]; M81dWb RCs from Oh et al. [74].
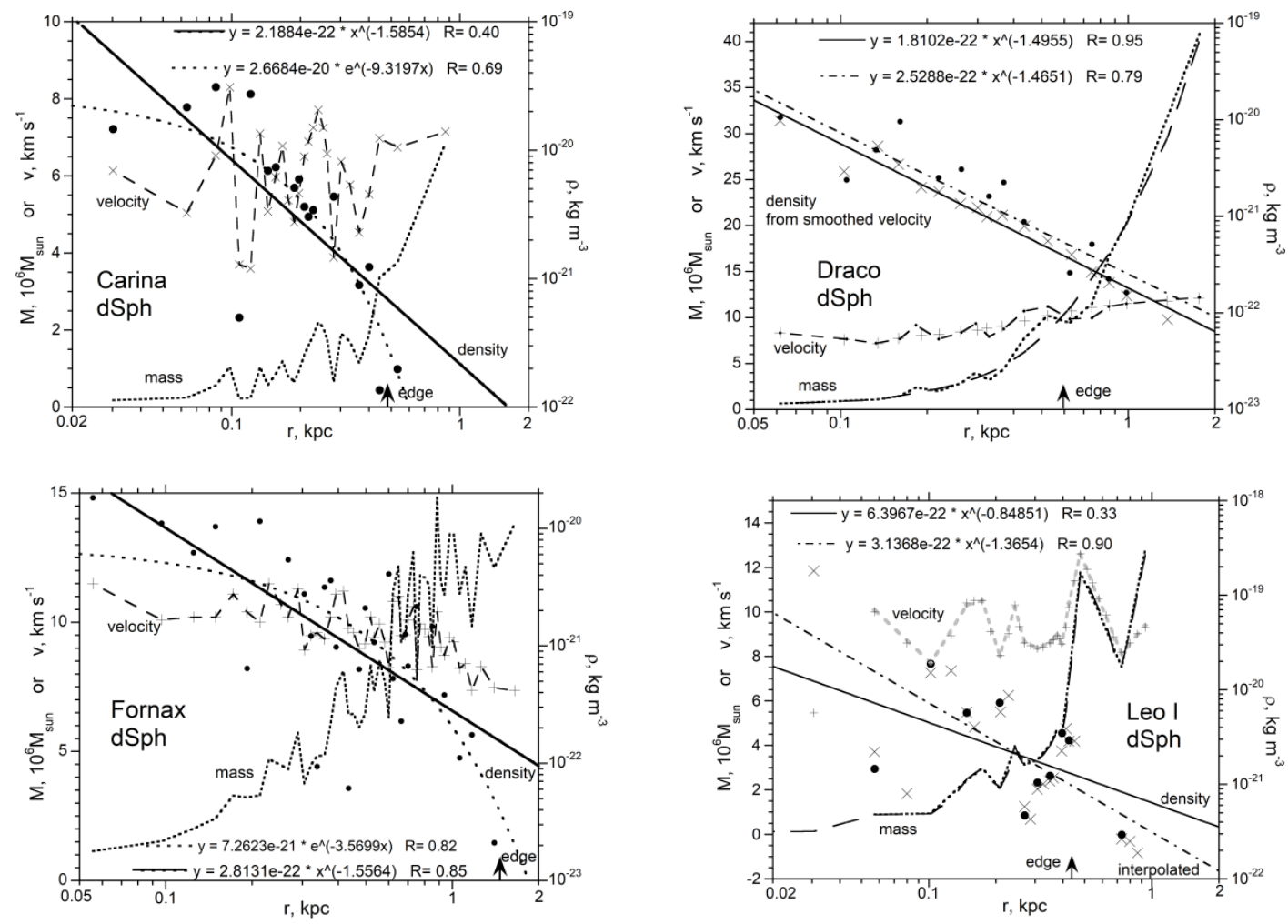

Figure A8. Dwarf spheroidal galaxies. RCs presented by Salucci et al. [75] were digitized. Original data from Walker et al. [76.77] and Mateo et al. [78]. Fornax is larger, more like the dwarf irregulars in Figure A7. For Draco, we compare extractions from the published data with those from the same data, but smoothed, and find little effect on the results. For Leo I, we compare extractions from the published data with those for an interpolated data set. Interpolating at $r$ below the lowest measurement is equivocal and the choice of $\mathrm{v}$ affects interior density. 

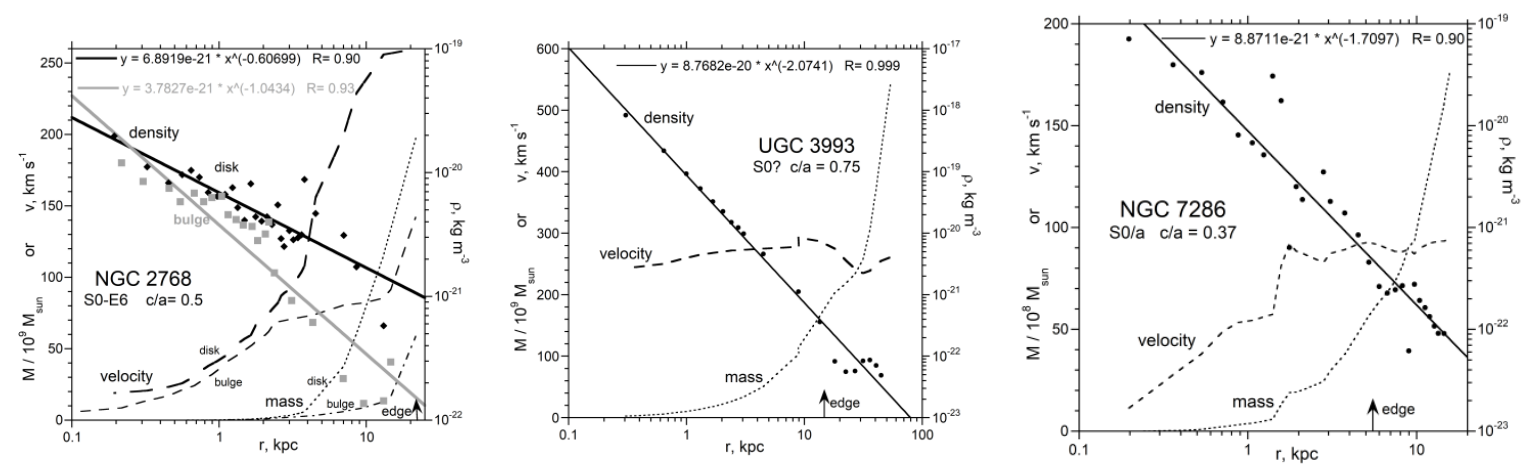

Figure A9. Lenticular galaxies. RCs of UGC 3993 and NGC 7786 from Noordemeer et al. [61]. RCs of NGC 2768 from Forbes et al. [42].
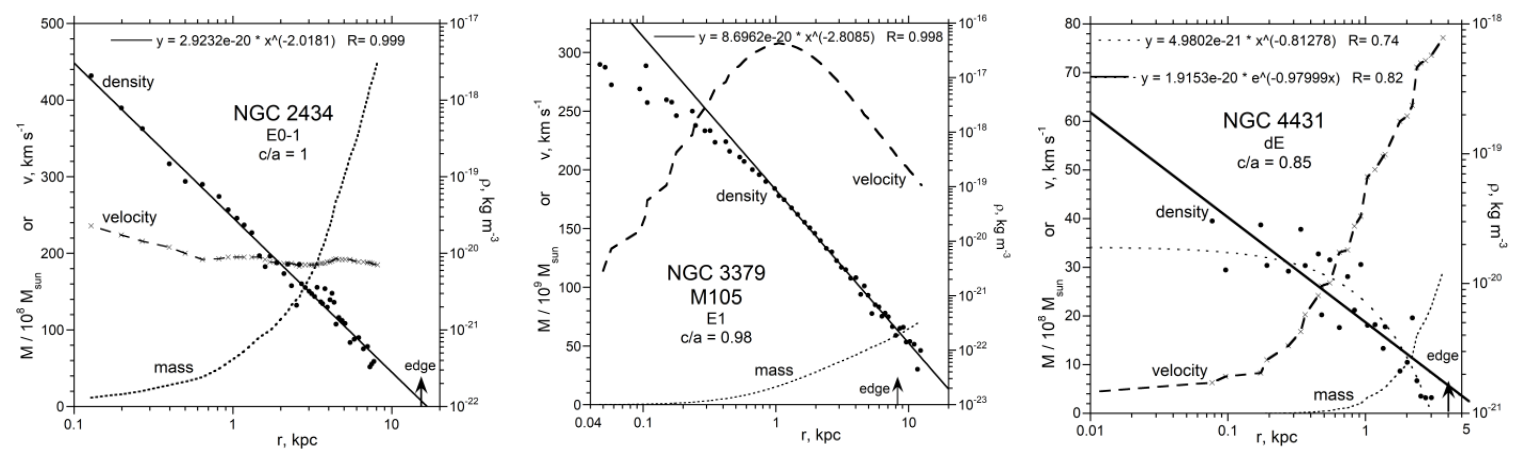

Figure A10. Elliptical galaxies. RCs of nearly spherical NGC 2434 from Rix et al. [68]. RCs of dwarf NGC 4431 from Toluba et al. [79]. RCs of NCG 3379 from Romanowsky et al. [31].

\section{References}

1. Kellogg, O.D. Foundations of Potential Theory; Dover Publications: New York, NY, USA, 1953.

2. Schmidt, M. A model of the distribution of mass in the galactic system. Bul. Astron. Inst. Neth. 1956, 13, 15.

3. McCarthy, J.E.; Yu Backhaus, E.; Fajans, J. Solving Poisson's equation with interior conditions. J. Math. Phys. 1998, 239, 6720. [CrossRef]

4. Hofmeister, A.M.; Criss, R.E.; Criss, E.M. Verified solutions for the gravitational attraction to an oblate spheroid: Implications for planet mass and satellite orbits. Planet. Space Sci. 2018, 152, 68-81. [CrossRef]

5. Hofmeister, A.M.; Criss, R.E. The physics of galactic spin. Can. J. Phys. 2017, 95, 156-166. [CrossRef]

6. Hofmeister, A.M.; Criss, R.E. Implications of geometry and the theorem of Gauss on Newtonian gravitational systems and a caveat regarding Poisson's equation. Galaxies 2017, 5, 89-100. [CrossRef]

7. Halliday, D.; Resnick, R. Physics; John Wiley and Sons: New York, NY, USA, 1966.

8. Diaz, R.A.; Herrera, W.J.; Martinez, R. Moments of inertia for solids of revolution and variational methods. Eur. J. Phys. 2006, 27, 183-192. [CrossRef]

9. Hofmeister, A.M.; Criss, R.E. Spatial and symmetry constraints as the basis of the Virial Theorem and astrophysical implications. Can. J. Phys. 2016, 94, 380-388. [CrossRef]

10. Rubin, V.C.; Ford, W.K. Rotation of the Andromeda nebula from a spectroscopic survey of emission regions. Astrophys. J. 1970, 159, 379-403. [CrossRef]

11. Faber, S.M.; Gallagher, J.S. Masses and mass-to-light ratios of galaxies. Ann. Rev. Astron. Astrophys. 1979, 17, 135-187. [CrossRef]

12. Dehnen, W.; Binney, J. Mass models of the Milky Way. Mon. Not. R. Astron. Soc. 1998, 294, 429-438. [CrossRef]

13. Ibata, R.; Lewis, G.F.; Martin, N.F.; Bellazzini, M.; Correnti, M. Does the Sagittarius stream constrain the Milky Way halo to be triaxial? Astrophys. J. Lett. 2013, 765, L155. [CrossRef]

14. Kam, Z.S.; Carignan, C.; Chemin, L.; Amram, P.; Epinat, B. Kinematics and mass modelling of M33, H $\alpha$ observations. Mon. Not. R. Astron. Soc. 2015, 449, 4048-4070. [CrossRef] 
15. Fuks, D.B.; Tabachnikov, S. Mathematical Omnibus: Thirty Lectures on Classic Mathematics; American Mathematical Society: Providence, RI, USA, 2007; pp. 417-418.

16. Doglioni, C.; Panza, G. Polarized plate tectonics. Adv. Geophys. 2015, 56, 1-167.

17. Zhang, J.; Song, X.; Li, Y.; Richards, P.; Sun, X.; Waldhauser, F. Inner core differential motion confirmed by earthquake waveform doublets. Science 2005, 309, 1357-1359. [CrossRef] [PubMed]

18. Criss, R.E. Analytics of planetary rotation: Improved physics with implications for the shape and super-rotation of Earth's Core. Earth Sci. Rev. 2019, 192, 471-479. [CrossRef]

19. Dufton, P.L.; Dunstall, P.R.; Evans, C.J.; Brott, I.; Cantiello, M.; de Koter, A.; de Mink, S.E.; Fraser, M.; Hénault-Brunet, V.; Howarth, I.D.; et al. The VLT-FLAMES tarantula survey: The fastest rotating O-type star and shortest period LMC pulsar-Remnants of a supernova disrupted binary? Astrophys. J. 2011, 743, L22:1-L22:6. [CrossRef]

20. Todhunter, I. A History of the Mathematical Theories of Attraction and Figure of the Earth; MacMillan and Co.: London, UK, 1873; reprinted in Dover Publications: New York, NY, USA, 1962.

21. Wiegert, T.; Irwin, J.; Miskolczi, A.; Schmidt, P.; Carolina Mora, S.; Damas-Segovia, A.; Stein, Y.; English, J.; Rand, R.J.; Santistevan, I. CHANG-ES. IV. Radio continuum emission of 35 edge-on galaxies observed with the Karl G. Jansky very large array in D configuration-Data release 1. Astronom. J. 2015, 150, 81. [CrossRef]

22. CHANG-ES Continuum Halos in Nearby Galaxies- and EVLA Survey. Available online: http://www.queensu. $\mathrm{ca} /$ changes (accessed on 26 January 2020).

23. NASA/IPAC Extragalactic Database. Available online: https://ned.ipac.caltech.edu/ (accessed on 1 December 2015).

24. Carignan, C. Light and mass distribution of the magellanic-type spiral NGC 3109. Astrophys. J. 1985, 299, 59-73. [CrossRef]

25. Jarrett, T.H.; Chester, T.; Cutri, R.; Schneider, S.E.; Huchra, J.P. The 2MASS Large Galaxy Atlas. Astron. J. 2003, 125, 525-554. [CrossRef]

26. Gadotti, D.A.; Sánchez-Janssen, R. Surprises in image decomposition of edge-on galaxies: Does Sombrero have a (classical) bulge? Mon. Not. R. Ast. Soc. 2012, 423, 877. [CrossRef]

27. Criss, R.E.; Hofmeister, A.M. Galactic density and evolution based on the virial theorem, energy minimization, and conservation of angular momentum. Galaxies 2018, 6, 115-135. [CrossRef]

28. Moulton, F.R. An Introduction to Celestial Mechanics; MacMillan: New York, NY, USA, 1914.

29. Burbidge, E.M.; Burbidge, G.R.; Prendergast, K.H. The rotation and mass of NGC 2146. Astrophys. J. 1959, 130, 739-748. [CrossRef]

30. Emden, R. Gaskuglen—Anwendungen de Mechanischen Wärmetheorie; Teubner, B.G.: Leipzig, Germany, 1907.

31. Romanowsky, A.J.; Douglas, N.G.; Arnaboldi, M.; Kuijken, K.; Merrifield, M.R.; Napolitano, N.R.; Capaccioli, M.; Freeman, K. A dearth of dark matter in ordinary elliptical galaxies. Science 2003, 301, 1696-1698. [CrossRef]

32. LeDrew, G. The real starry sky. J. R. Astron. Soc. Can. 2011, 95, 322-324.

33. Luyten, W.J. A new determination of the luminosity function. Mon. Not. R. Astron. Soc. 1968, 139, $221-224$. [CrossRef]

34. Ferrière, K. The interstellar environment of our galaxy. Rev. Mod. Phys. 2011, 73, 1031. [CrossRef]

35. Fang, T.; Buote, D.A.; Humphrey, P.J.; Canizares, C.R.; Zappacosta, L.; Maiolino, R.; Tagliaferri, G.; Gastaldello, F. Confirmation of X-ray absorption by warm-hot intergalactic medium in the Sculptor wall. Astrophys. J. 2010, 714, 1715-1724. [CrossRef]

36. Outer Space. Available online: https://en.wikipedia.org/wiki/Outer_space (accessed on 1 January 2016).

37. Craig, I.J.D.; Brown, J.C. Inverse Problems in Astronomy; Adam Hilger Ltd.: Bristol, UK, 1986.

38. Groetsch, C.W. Inverse Problems: Activities for Undergraduates; Cambridge University Press: Cambridge, UK, 1999.

39. Ambartsumian, V. On the derivation of the frequency function of space velocities of the stars from the observed radial velocities. Mon. Not. Roy. Astron. Soc. 1936, 96, 172. [CrossRef]

40. Sofue, Y.; Reuter, H.-P.; Krause, M.; Wielebinski, R.; Nakai, N. Peculiar rotations of molecular gas in M82-Keplerian disk and slowly rotating halo. Astrophys. J. 1992, 395, 126. [CrossRef]

41. Swaters, R.A.; Sancisi, R.; van der Hulst, J.M. The H I Halo of NGC 891. Astrophys. J. 1997, 491, 140. [CrossRef] 
42. Forbes, D.A.; Cortesi, A.; Pota, V.; Foster, C.; Romanowsky, A.J.; Merrifield, M.R.; Brodie, J.P.; Strader, J.; Coccato, L.; Napolitano, N. Radially extended kinematics in the S0 galaxy NGC 2768 from planetary nebulae, globular clusters and starlight. Mon. Not. R. Astron. Soc. 2012, 426, 975. [CrossRef]

43. Finkelman, I.; Funes, J.G.; Brosch, N. Polar ring galaxies in the Galaxy Zoo. Mon. Not. Royal Ast. Soc. 2012, 422, 2386-2398. [CrossRef]

44. Binney, J.; Tremaine, S. Galactic Dynamics, 2nd ed.; Princeton University Press: Princeton, NJ, USA, 2008.

45. Velázquez, J.J.L. Singularity formation for the Stephan problem. In Partial Differential Equations: Theory and Numerical Solution; Necas, J., Jager, W., Stara, J., John, O., Najzar, K., Eds.; Chapman and Hall: Boca Raton, FL, USA, 2000; pp. 312-335.

46. Arnold, V.I.; Kozlov, V.V.; Neishtadt, A.I. Mathematical Aspects of Classical and Celestial Mechanics; Springer: Berlin, Germany, 2006.

47. Sofue, Y.; Rubin, V.C. Rotation curves of spiral galaxies. Ann. Rev. Astron. Astrophys. 2001, 39, $137-174$. [CrossRef]

48. Wiegert, T.; English, J. Kinematic classification of non-interacting spiral galaxies. New Astron. 2014, $26,40-61$. [CrossRef]

49. Sersic, J.L. Extragalactic Astronomy. In Geophysics and Astrophysics Monographs, v. 20; D. Reidel Publishing Co.: Dordrecht, The Netherlands, 1982; p. 61.

50. Marr, J.H. Galaxy rotation curves with lognormal density distribution. Mon. Not. R. Astron. Soc. 2015, 448, 3229-3241. [CrossRef]

51. Brandt, J.C. On the distribution of mass in galaxies. I. The large-scale structure of ordinary spirals with applications to M31. Astrophys. J. 1960, 131, 293-303. [CrossRef]

52. Lambas, D.G.; Maddox, S.J.; Loveday, J. On the true shapes of galaxies. Mon. Not. Roy. Astron. Soc. 1992, 258, 404. [CrossRef]

53. Sofue, Y.; Koda, J.; Nakanishi, H.; Onodera, S. The Virgo high-resolution CO survey, II. Rotation curves and dynamical mass distributions. Publ. Astron. Soc. Jpn. 2003, 55, 59-74. [CrossRef]

54. De Blok, W.J.G.; Walter, F.; Brinks, E.; Trachternach, C.; Oh, S.-H.; Kennicutt, R.C., Jr. High-resolution rotation curves and galaxy mass models from THINGS. Astrophys. J. 2008, 136, 2648-2719. [CrossRef]

55. Howley, K.M.; Guhathakurta, P.; van der Marel, R.; Geha, M.; Kalirai, J.; Yniguez, B.; Kirby, E.; Cuillandre, J.-C.; Gilbert, K. Internal stellar kinematics of M32 from the SPLASH survey, dark halo constraints. Astrophys. J. 2013, 765, 65. [CrossRef]

56. Redshift Independent Distances (NED-D). Available online: https://ned.ipac.caltech.edu/Library/Distances/ (accessed on 31 January 2020).

57. Sofue, Y. Mass distribution and rotation curve in the galaxy. In Planets, Stars and Stellar Systems; Gilmore, G., Ed.; Springer: New York, NY, USA, 2013; p. 985.

58. Sofue, Y. Rotation curve and mass distribution in the galactic center-From black hole to entire galaxy. Pub. Astron. Soc. Japan 2013, 65, 118. [CrossRef]

59. Sofue, Y. Dark halos of M31 and the Milky Way. Publ. Astron. Soc. Jpn. 2015, 67, 759. [CrossRef]

60. Watkins, L.L.; Van Der Marel, R.P.; Sangmo, S.T.; Evans, N.W. Evidence for an intermediate-mass Milky Way from Gaia DR2 halo globular cluster motions. Astrophys. J. 2019, 873, 118. [CrossRef]

61. Noordermeer, E.; van der Hulst, J.M.; Sancisi, R.; Swaters, R.S.; van Albada, T.S. The mass distribution in early-type disc galaxies, declining rotation curves and correlations with optical properties. Mon. Not. R. Astron. Soc. 2007, 376, 513-546. [CrossRef]

62. Roelfsema, P.R.; Allen, R.J. Radio observations of H I in UGC 2885, the largest identified SC galaxy. Astron. Astrophys. 1985, 146, 213-222.

63. Greco, J.P.; Martini, P.; Thompson, T.A. Measurement of the mass and stellar population distribution in M82 with the LBT. Astrophys. J. 2012, 757, 24. [CrossRef]

64. Bottema, R.; Pestaña, J.L.G. The distribution of dark and luminous matter inferred from extended rotation curves. Mon. Not. R. Astron. Soc. 2015, 448, 2566-2593. [CrossRef]

65. Sofue, Y. Galaxy, ISM, and Radio Astronomy. Available online: http//www.ioa.s.u-tokyo.ac.jp/ \{\}sofue/ (accessed on 15 June 2015).

66. Sofue, Y.; Tutui, Y.; Honma, M.; Tomita, A.; Takamiya, T.; Koda, J.; Takeda, Y. Central rotation curves of spiral galaxies. Astrophys. J. 1999, 523, 136-146. [CrossRef] 
67. Pavlovich, K.; Pavlovich, A.; Sipols, A. Newtonian explanation of galaxy rotation curves based on distribution of baryonic matter. arXiv 2014, arXiv:1406.2401P.

68. Rix, H.-W.; de Zeeuw, P.T.; Cretton, N.; van der Marel, R.P.; Carollo, C.M. Dynamical modeling of velocity profiles, the dark halo around the elliptical galaxy NGC 2434. Astrophys. J. 1997, 488, 702. [CrossRef]

69. Hlavacek-Larrondo, J.; Carignan, C.; Daigle, O.; de Denus-Baillargeon, M.-M.; Marcelin, M.; Epinat, B.; Hernandez, O. Deep H alpha observations of NGC 253, a very extended and possibly declining rotation curve? Mon. Not. R. Ast. Soc. 2011, 411, 71-84. [CrossRef]

70. Corbelli, E.; Salucci, P. The extended rotation curve and the dark matter halo of M33. Mon. Not. R. Ast. Soc. 2000, 311, 441-447. [CrossRef]

71. Jardel, J.R.; Gebhardt, K.; Shen, J.; Fisher, D.B.; Kormendy, J.; Kinzler, J.; Lauer, T.R.; Richstone, D.; Gültekin, K. Orbit-based dynamical models of the Sombrero galaxy (NGC 4594). Astrophys. J. 2011, 739, 21. [CrossRef]

72. Kormendy, J.; Westphal, D.J. Noncircular gas velocities and the radial dependence of mass-to-light ratio in NGC 4594 (the Sombrero Galaxy). Astrophys. J. 1989, 338, 752-760. [CrossRef]

73. Leaman, R.; Venn, K.A.; Brooks, A.M.; Battaglia, G.; Cole, A.A.; Ibata, R.A.; Irwin, M.J.; McConnachie, A.W.; Mendel, J.T.; Tolstoy, E. The resolved structure and dynamics of an isolated dwarf galaxy, A VLT and Keck spectroscopic survey of WLM. Astrophys. J. 2012, 750, 33. [CrossRef]

74. Oh, S.-H.; de Blok, W.J.G.; Walter, F.; Brinks, E.; Kennicutt, R.C., Jr. High-resolution dark matter density profiles of THINGS dwarf galaxies, correcting for noncircular motions. Astron. J. 2008, 136, 2761-2781. [CrossRef]

75. Salucci, P.; Wilkinson, M.I.; Walker, M.G.; Gilmore, G.F.; Grebel, E.K.; Koch, A.; Frigerio Martins, C.; Wyse, R.F.G. Dwarf spheroidal galaxy kinematics and spiral galaxy scaling laws. Mon. Not. R. Ast. Soc. 2012, 420, 2034. [CrossRef]

76. Walker, M.G.; Mateo, M.; Olszewski, E.W.; Gnedin, O.Y.; Wang, X.; Sen, B.; Woodroofe, M. Velocity dispersion profiles of seven dwarf spheroidal galaxies. Astrophys. J. 2007, 667, L53. [CrossRef]

77. Walker, M.G.; Mateo, M.; Olszewski, E.W. Stellar velocities in the Carina, Fornax, Sculptor, and Sextans dSph galaxies, data from the Magellan/MMFS survey. Astronom. J. 2009, 137, 3100. [CrossRef]

78. Mateo, M.; Olszewski, E.W.; Walker, M.G. The velocity dispersion profile of the remote dwarf spheroidal galaxy Leo I, A tidal hit and run? Astrophys. J. 2008, 675, 20. [CrossRef]

79. Toloba, E.; Guhathakurta, P.; Peletier, R.F.; Boselli, A.; Lisker, T.; Falcón-Barroso, J.; Simon, J.D.; van de Ven, G.; Paudel, S.; Emsellem, E.; et al. Stellar kinematics and structural properties of Virgo cluster dwarf early-type galaxies from the SMAKCED Project. II. The survey and a systematic analysis of kinematic anomalies and asymmetries. Astrophys. J. Suppl. 2014, 215, 17. [CrossRef]

80. Iodice, E.; Coccato, L.; Combes, F.; de Zeeuw, T.; Arnaboldi, M.; Weilbacher, P.M.; Bacon, R.; Kuntschner, H.; Spavone, M. Mapping the inner regions of the polar disk galaxy NGC 4650A with MUSE. Astron. Astrophys. 2015, 583, A48. [CrossRef]

81. Honma, M.; Nagayama, T.; Ando, K.; Bushimata, T.; Choi, Y.K.; Handa, T.; Hirota, T.; Imai, H.; Jike, T.; Kim, M.K.; et al. Fundamental parameters of the Milky Way Galaxy based on VLBI astrometry. Pub. Astr. Soc. Japan 2012, 64, 136. [CrossRef]

82. Nakanishi, H.; Sakai, N.; Kurayama, T.; Matsuo, M.; Imai, H.; Burns, R.A.; Ozawa, T.; Honma, M.; Shibata, K.; Kawaguchi, N. Outer rotation curve of the Galaxy with VERA. II. Annual parallax and proper motion of the star-forming region IRAS 21379+5106. Pub. Astr. Soc. Jpn. 2015, 67, 6813. [CrossRef]

83. Mateo, M.L. Dwarf galaxies of the Local Group. Ann. Rev. Astron. Astrophys. 1998, 36, 435-506. [CrossRef]

84. Pawlowski, M.S.; Pflamm-Altenburg, J.; Kroupa, P. The VPOS: A vast polar structure of satellite galaxies, globular clusters and streams around the Milky Way. Mon. Not. R. Ast. Soc. 2012, 423, 1109-1126. [CrossRef]

85. Bureau, M.; Carignan, C. Environment, RAM pressure, and shell formation in Holmberg II. Astron. J. 2002, 123, 1316. [CrossRef]

86. Khoperskov, S.A.; Moiseev, A.V.; Khoperskov, A.V.; Saburova, A.S. To be or not to be oblate: The shape of the dark matter halo in polar ring galaxies. Mon. Not. R. Ast. Soc. 2014, 441, 2650-2662. [CrossRef]

87. Lehner, N.; Howk, J.C.; Wakker, B.P. Evidence for a massive, extended circumgalactic medium around the Andromeda galaxy. Astrophys. J. 2015, 804, 79. [CrossRef]

88. Boroson, T. The distribution of luminosity in spiral galaxies. Astrophys. J. Supp. 1981, 46, 177. [CrossRef] 
89. Sparke, L.S.; Gallagher, J.S. Galaxies in the Universe; Cambridge University Press: Cambridge, UK, 2000.

90. Zombeck, M.V. Handbook of Space Astronomy and Astrophysics; Cambridge Univ. Press: Cambridge, UK, 2007.

91. Disney, M.J.; Romano, J.D.; Garcia-Appadoo, D.A.; West, A.A.; Dalcanton, J.J.; Cortese, L. Galaxies appear simpler than expected. Nature 2008, 455, 1082-1084. [CrossRef] [PubMed] 